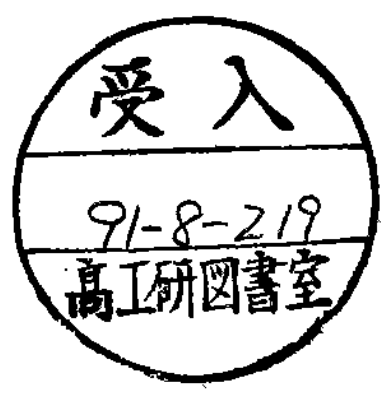

CERN-TH. $6124 / 91$

\title{
TOWARD A MODEL-INDEPENDENT ANALYSIS OF ELECTROWEAK DATA
}

\author{
G. Altarelli \\ CERN - Geneva \\ R. Barbieri \\ Dipartimento di Fisica, Università di Pisa \\ and \\ S. Jadach \\ CERN - Geneva
}

\begin{abstract}
We set the framework for a model-independent analysis of the data on electroweak precision tests. Starting from three basic observables, the mass ratio $\mathrm{m}_{\mathrm{W}} / \mathrm{m}_{\mathrm{Z}}$, the $\mathrm{Z}$ partial width and the forward-backward asymmetry for charged leptons, we define three dimensionless parameters $\varepsilon_{1}, \varepsilon_{2}$ and $\varepsilon_{3}$ which contain the small radiative correction effects one is interested in, with large $\mathrm{m}_{\mathrm{t}}$ effects only appearing in $\varepsilon_{1}$. The results on the epsilons implied by the present experimental data are discussed as well as the predictions of the Standard Model, as functions of $m_{t}$ and $m_{H}$, with special attention to evaluating the theoretical errors. We formulate a hierarchy of simple and general assumptions, valid in large classes of models, which are needed in order to relate the epsilons to an increasingly larger set of observables including the $\tau$-polarisation asymmetry, the forward-backward asymmetry for the b-quark, deep inelastic neutrino scattering and atomic parity violation. Correspondingly the analysis of present data is performed in stages and the conclusions are examined at each stage. Finally the case of the Standard Model is recovered as a very relevant particular example.
\end{abstract}

CERN-TH.6124/91

June 1991 


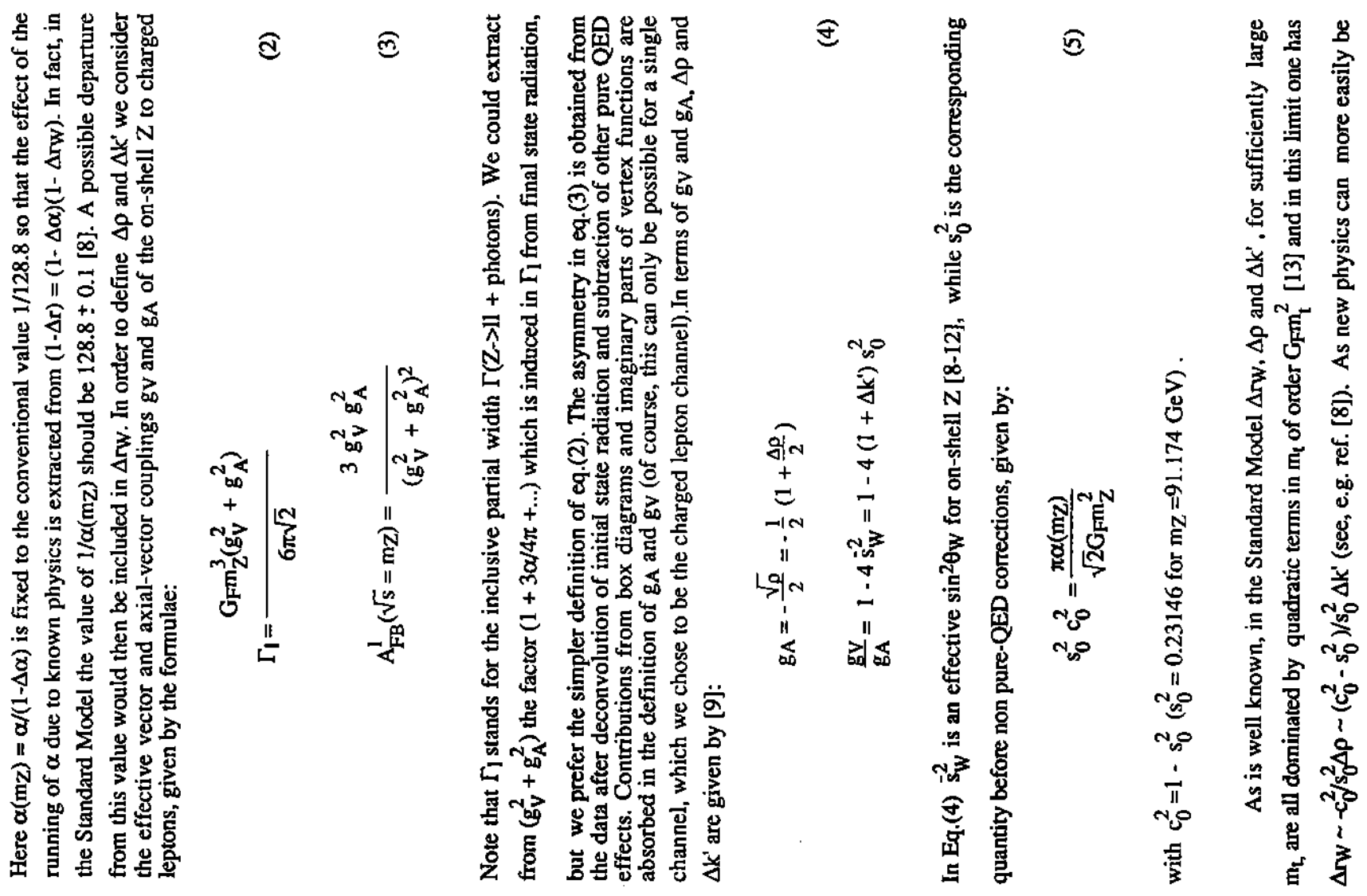

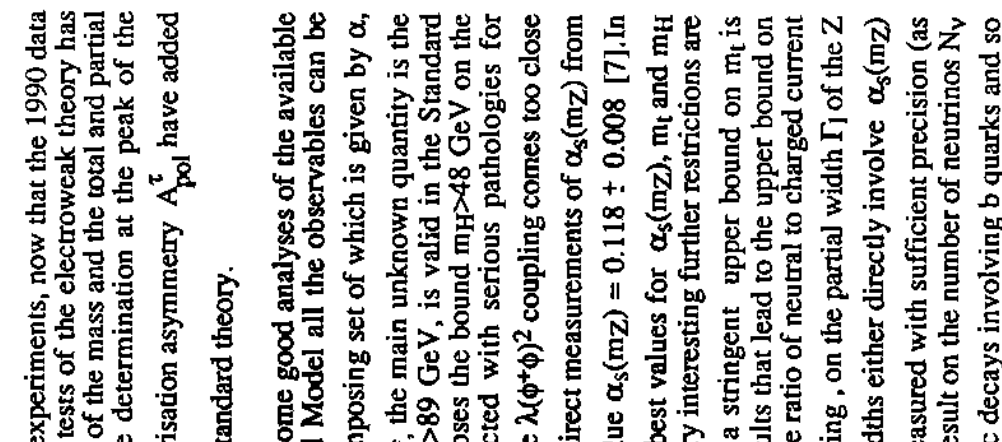

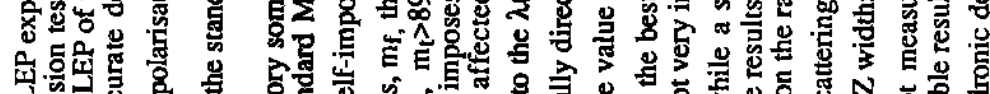
部

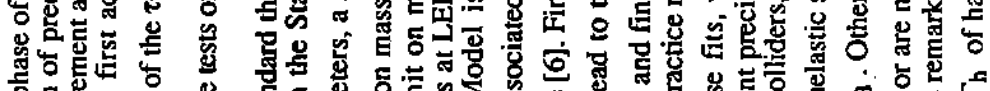

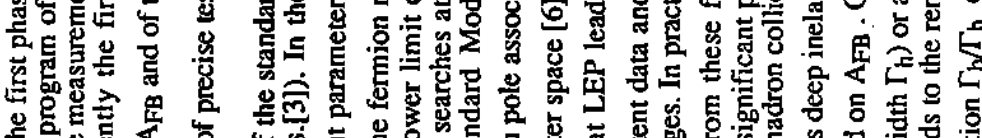
s. ste

5大亏

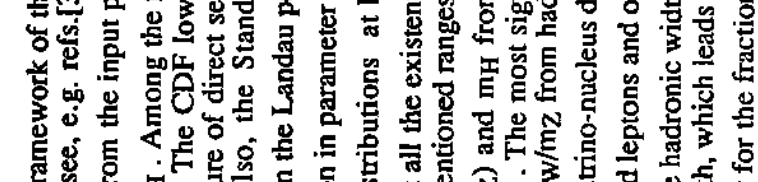

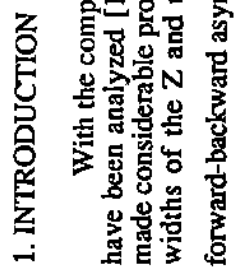

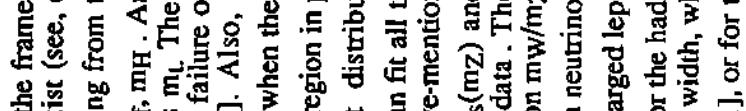

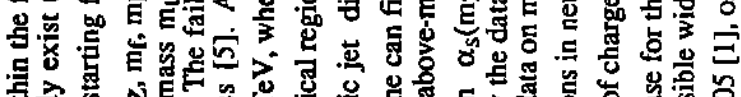

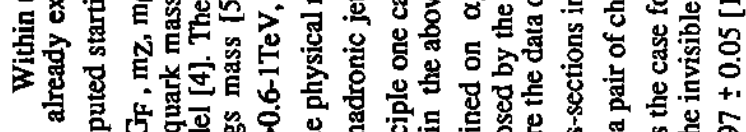

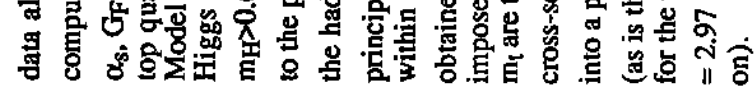

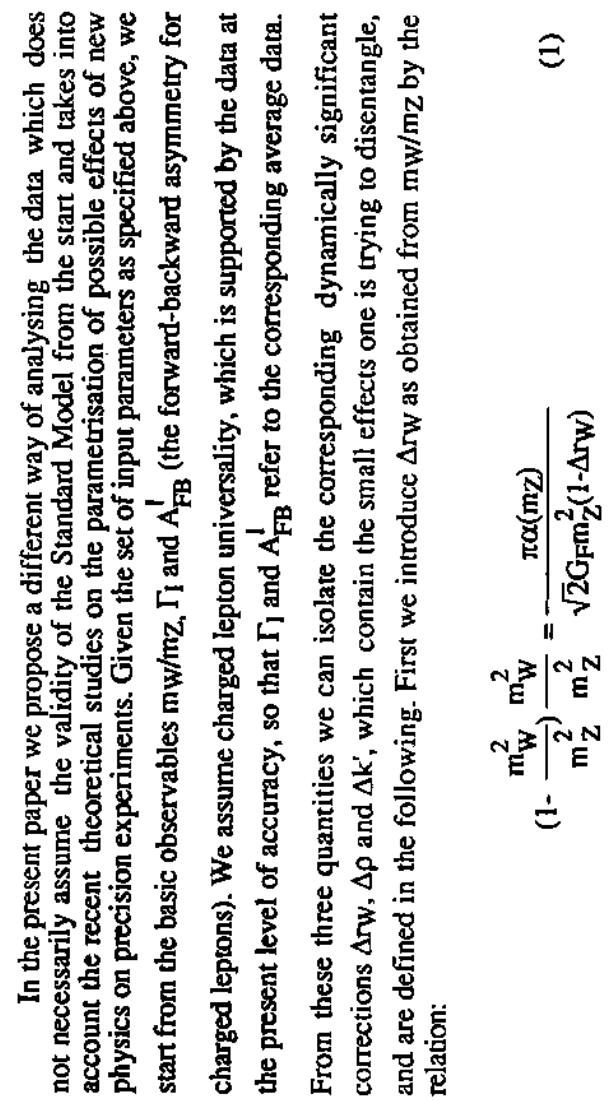



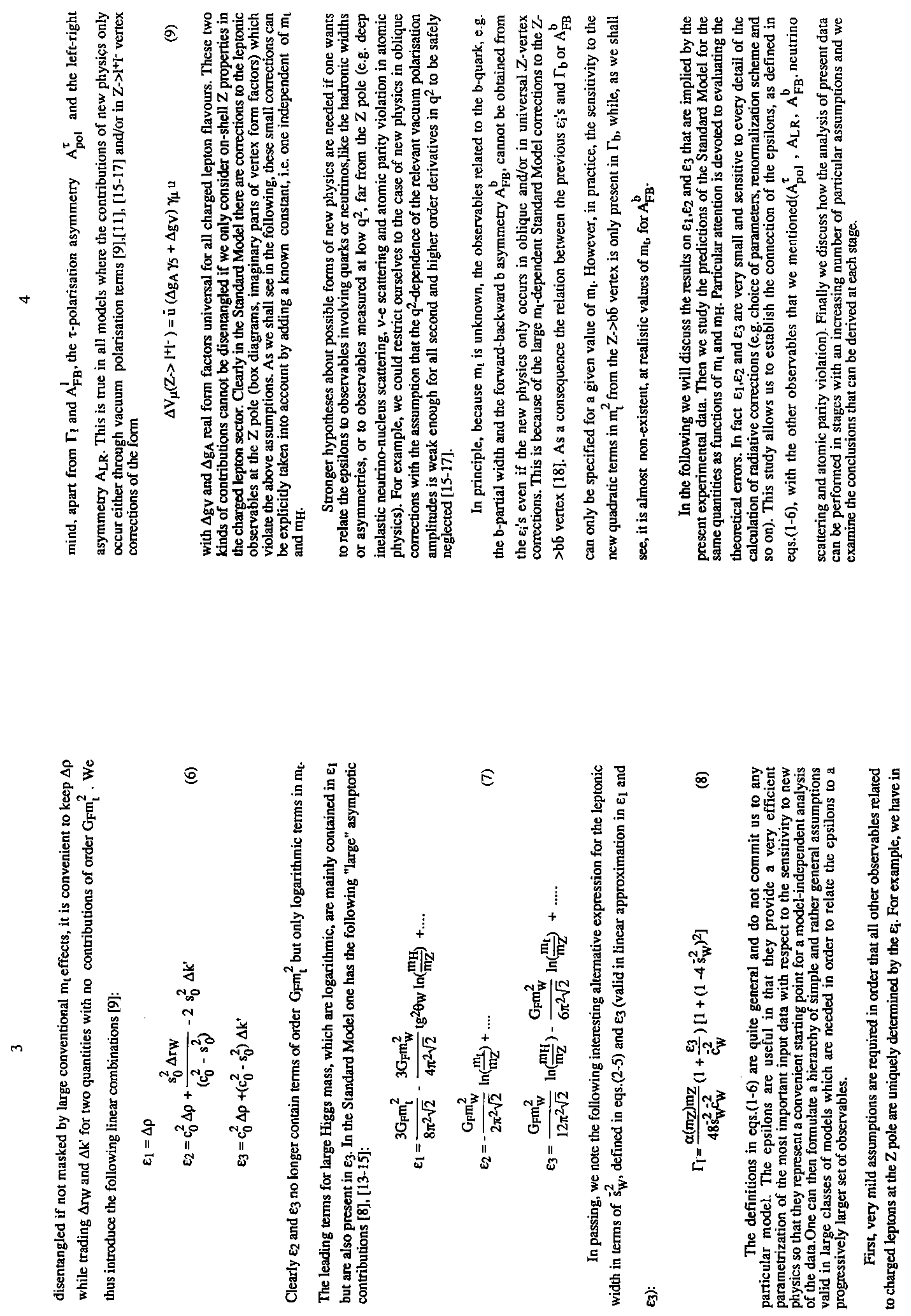

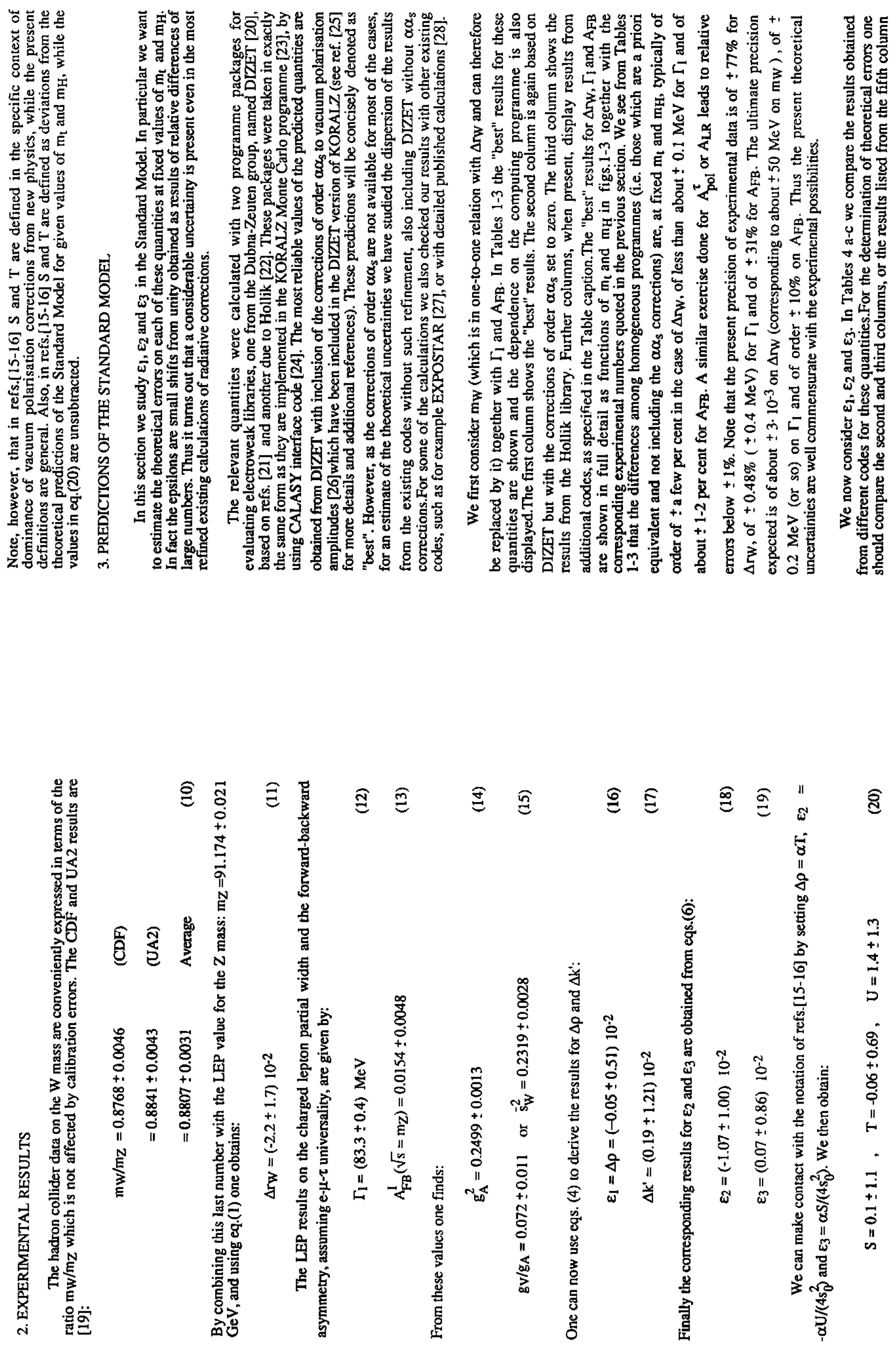


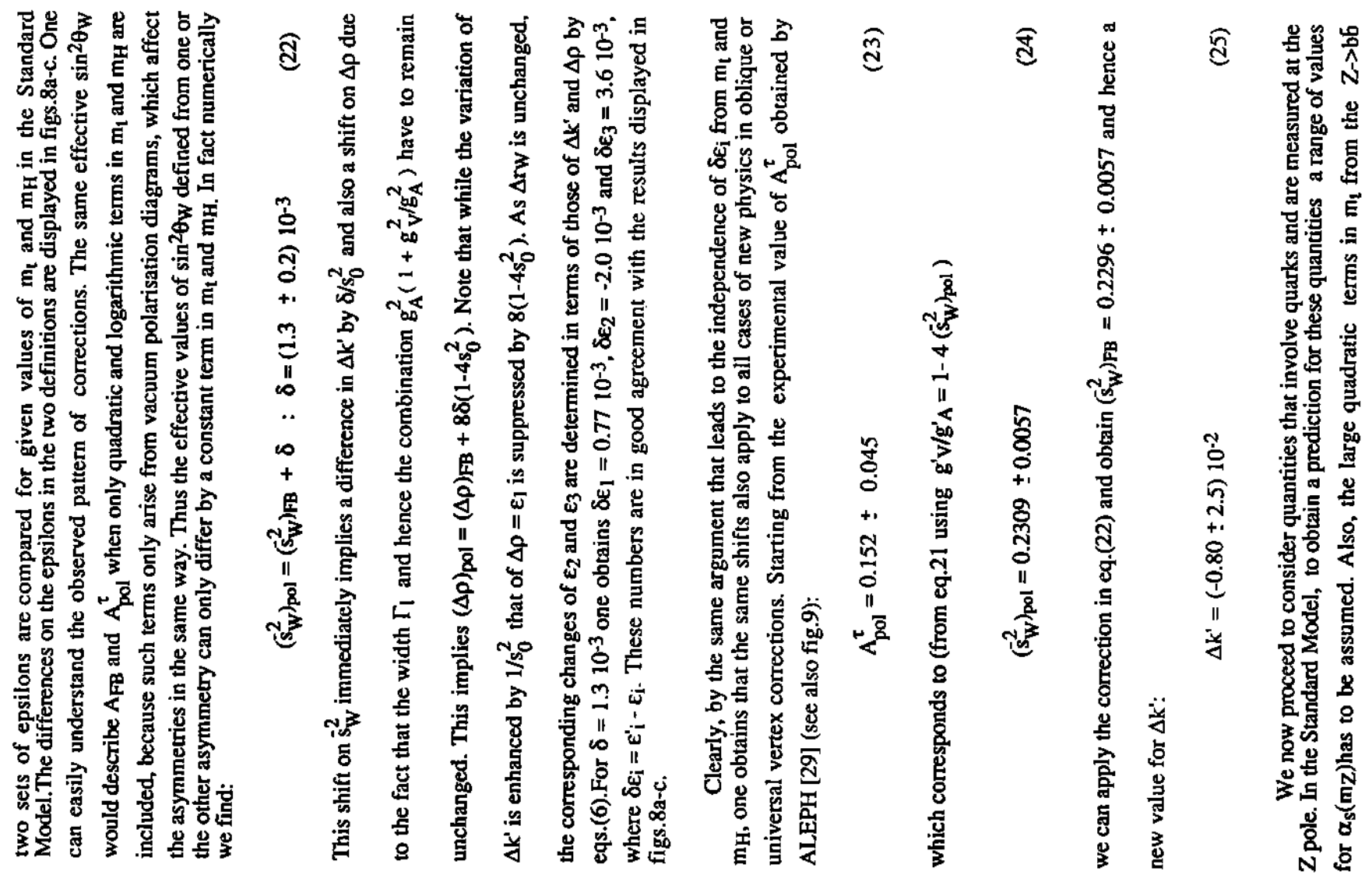

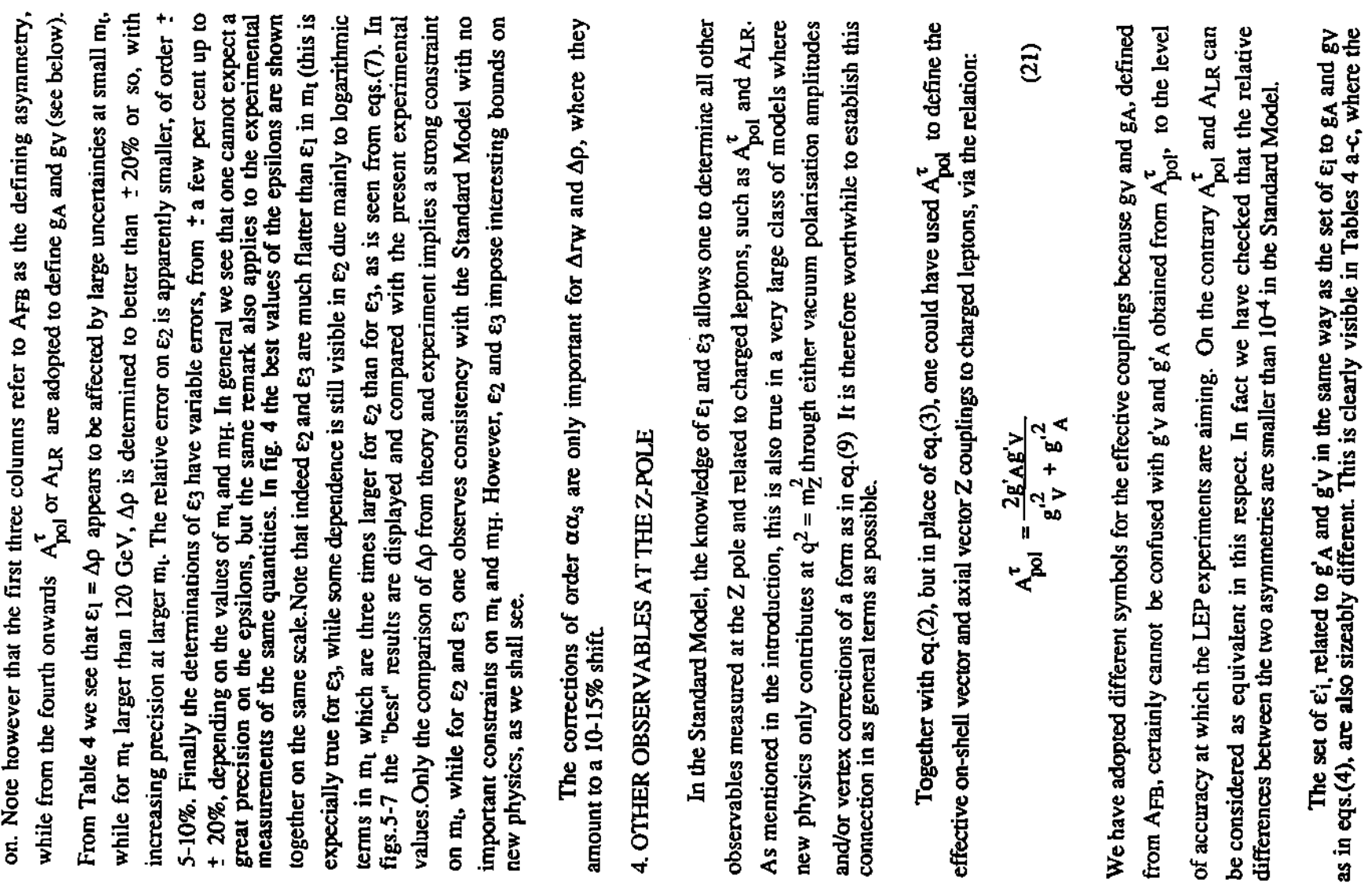




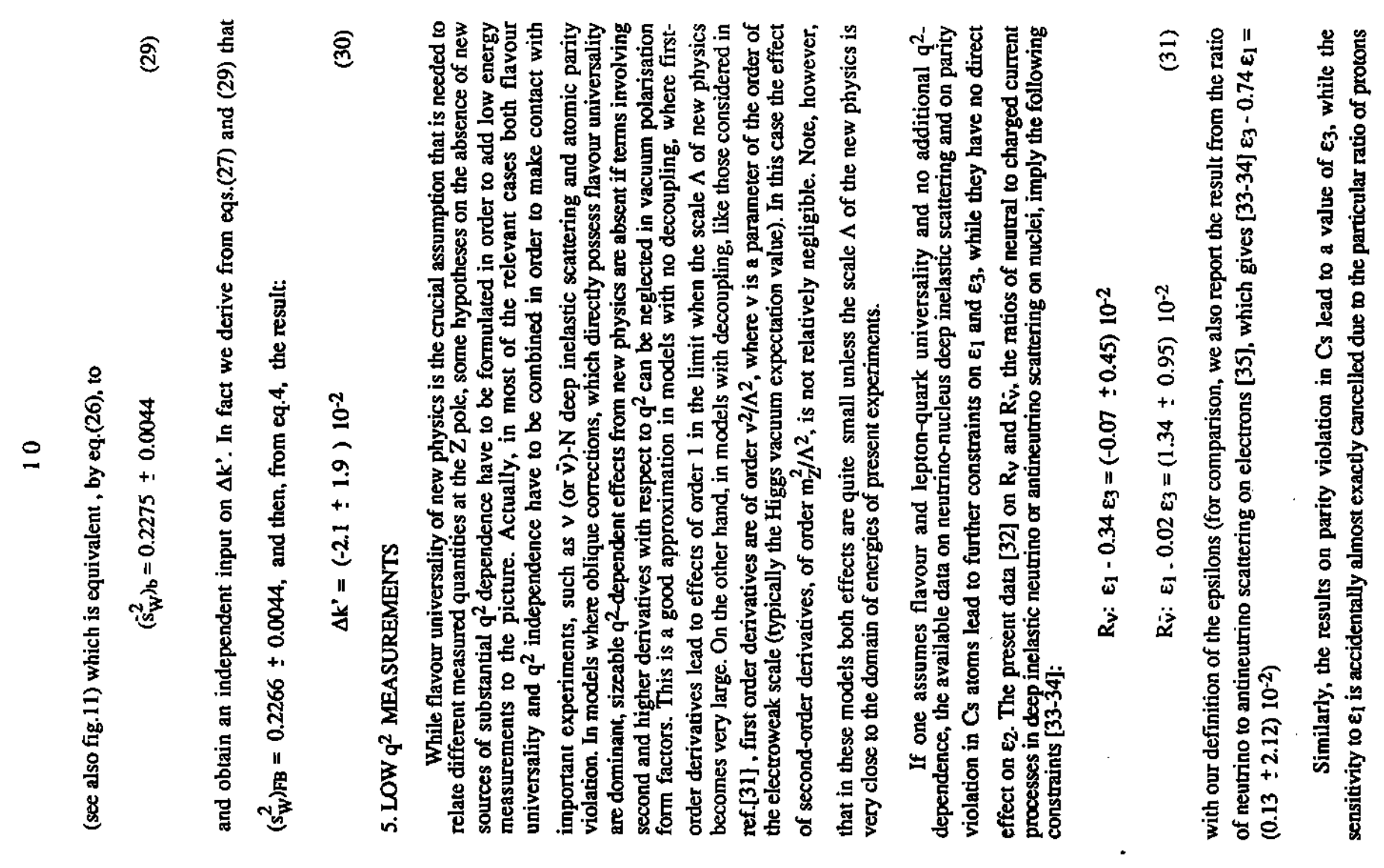

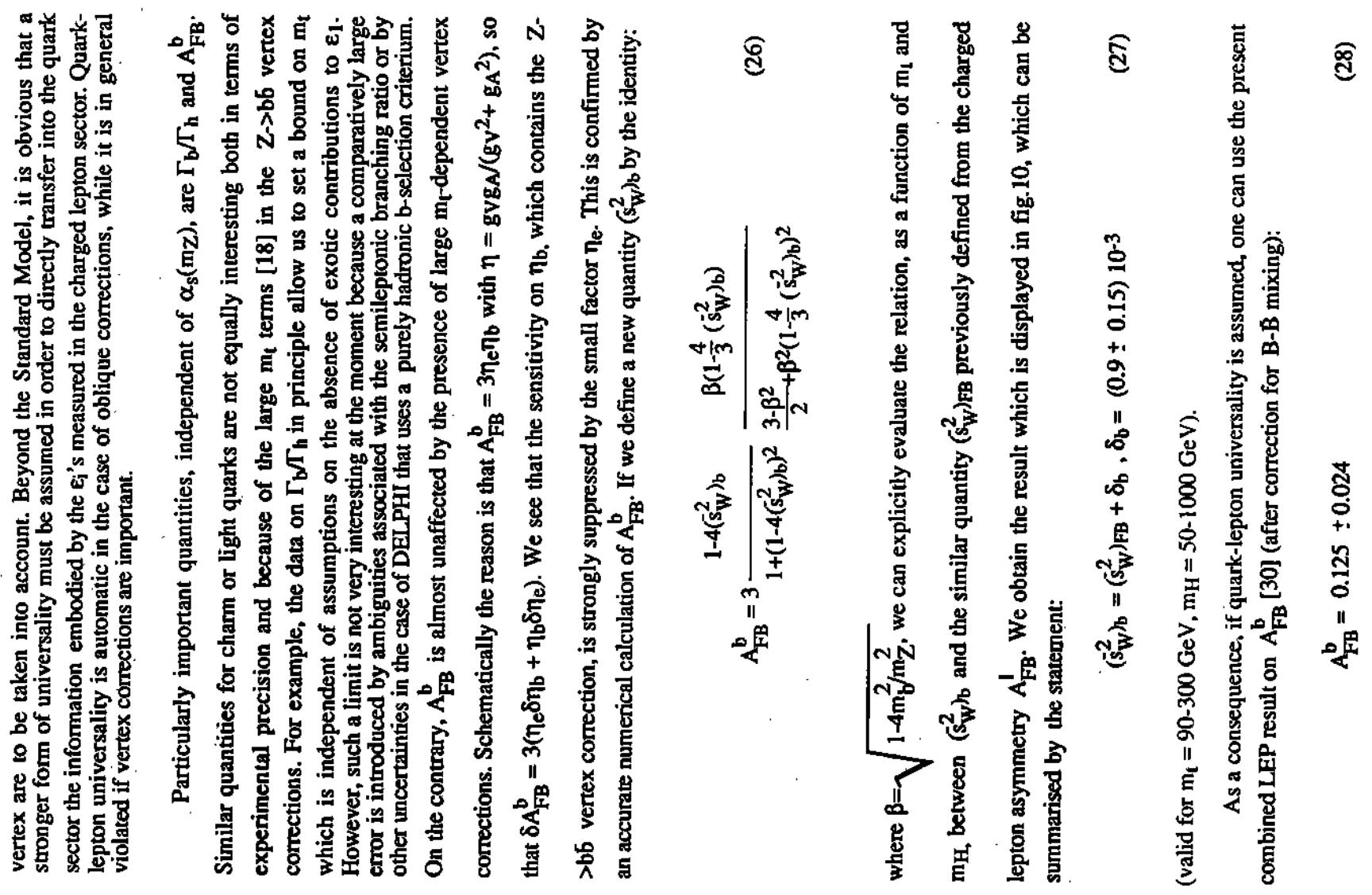



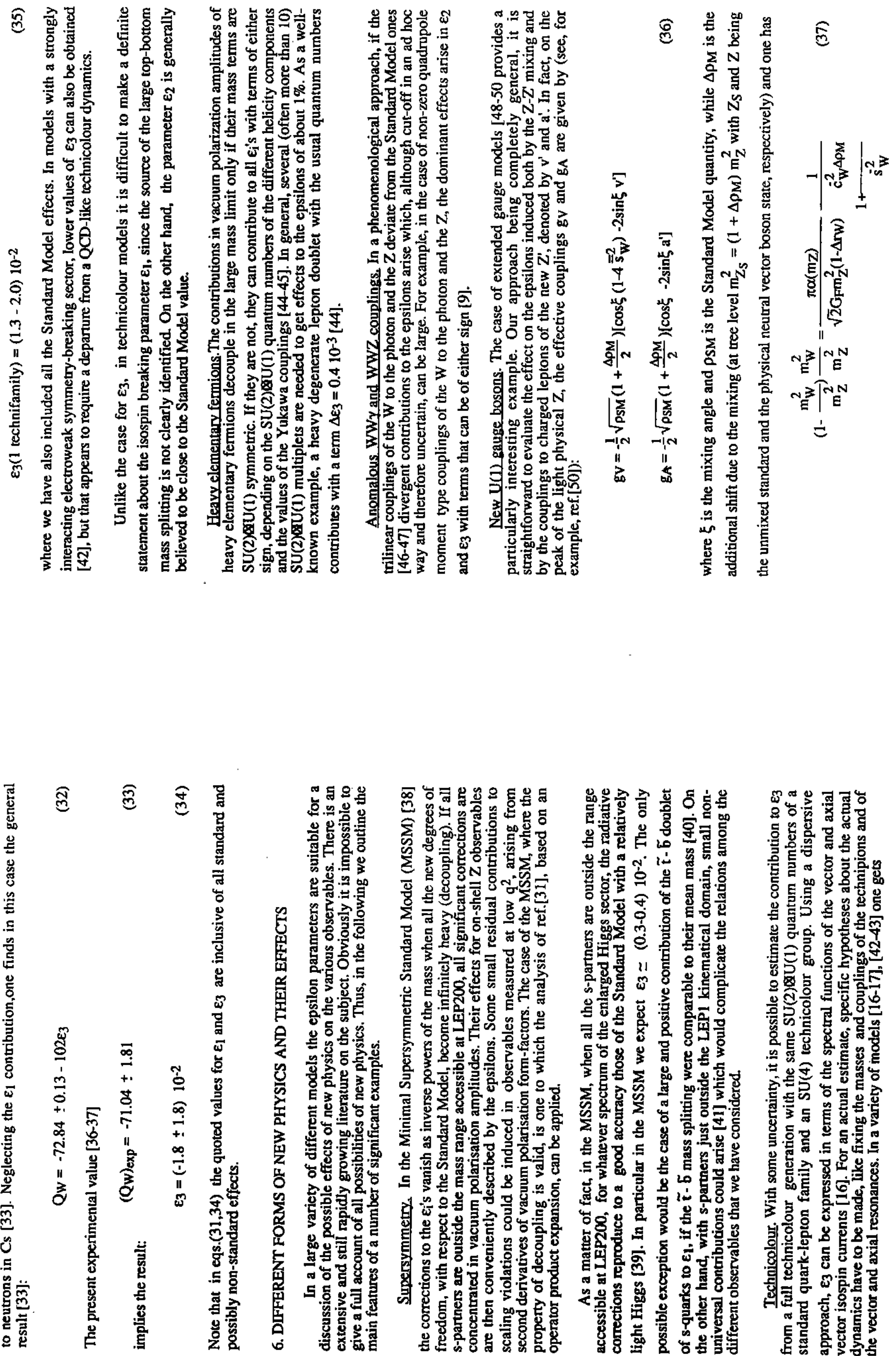


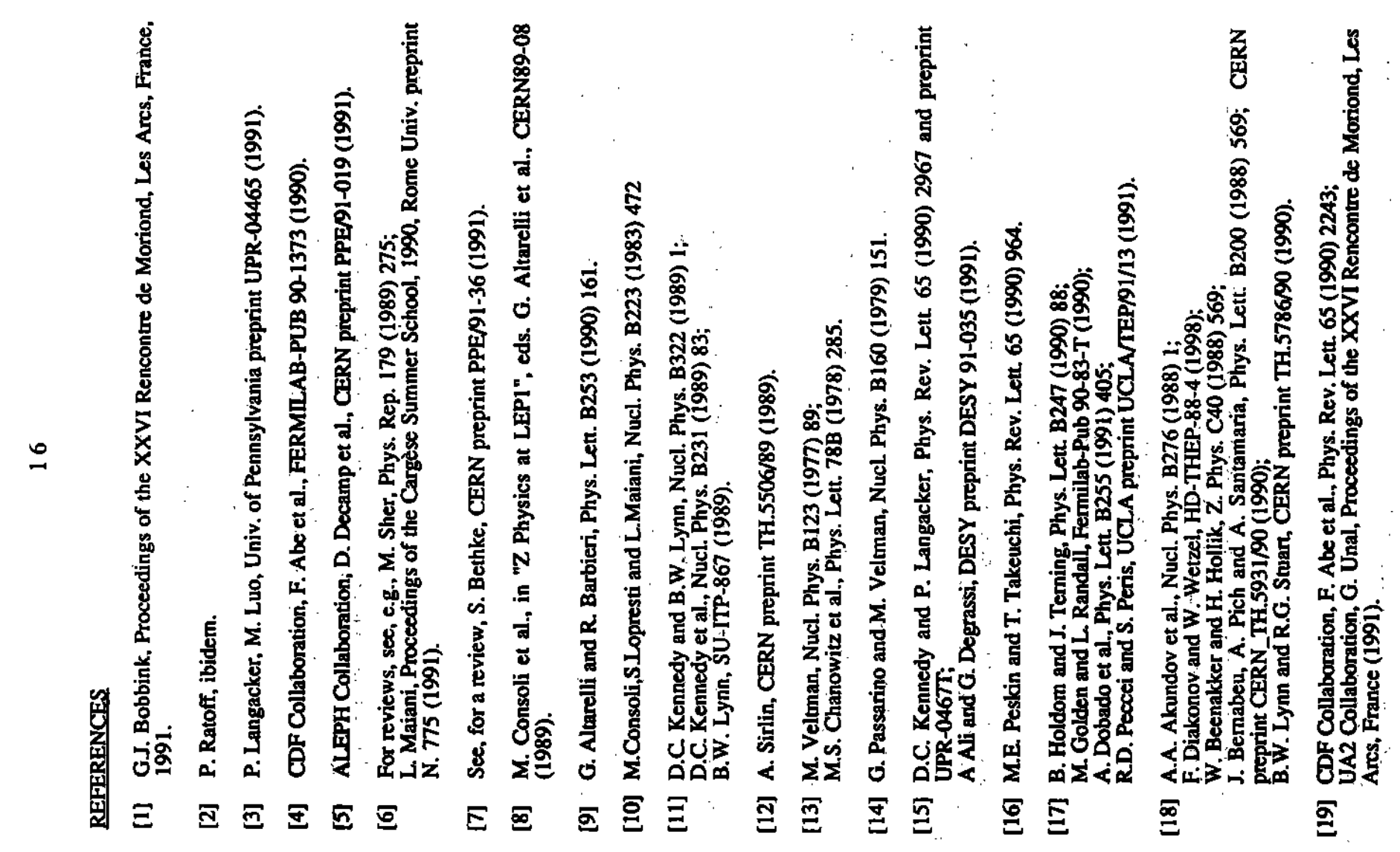

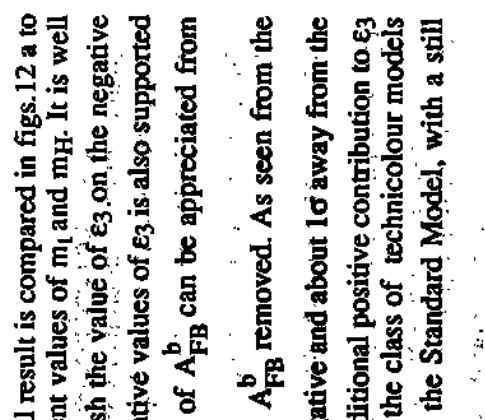

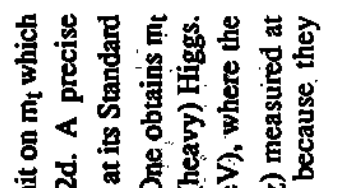

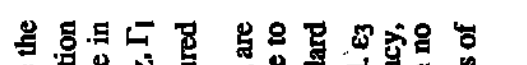

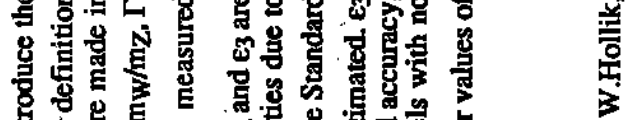

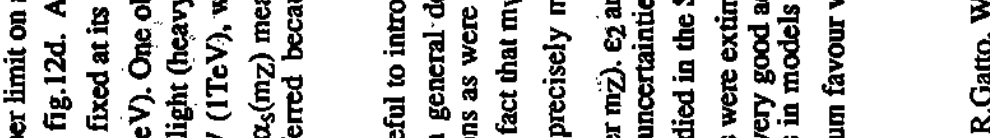

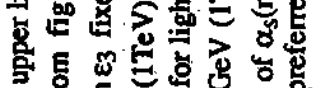

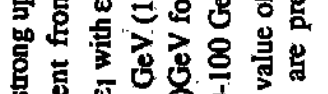

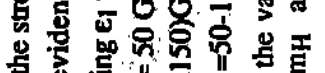

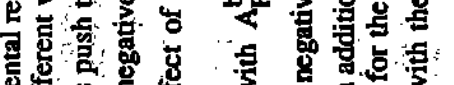

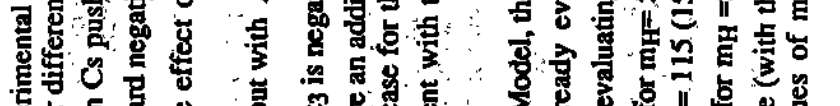

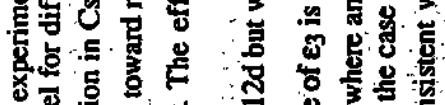

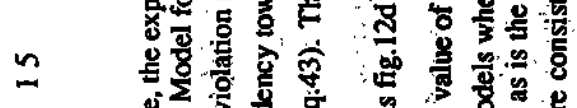

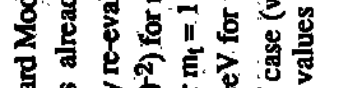

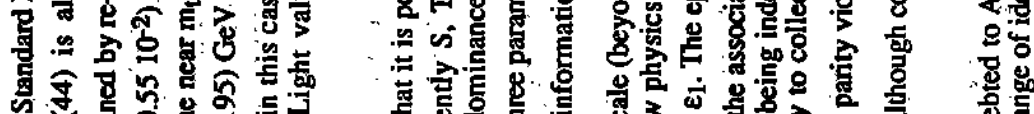

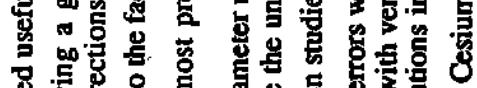

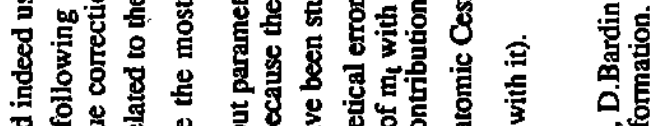

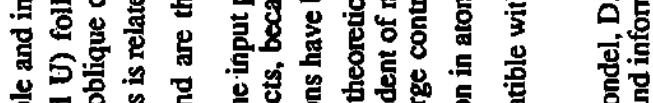

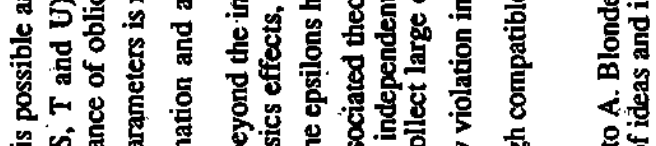

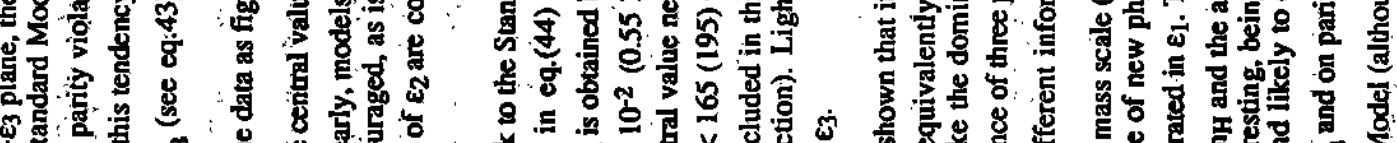

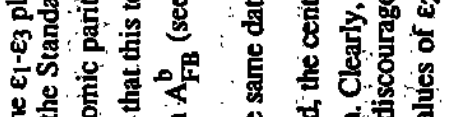

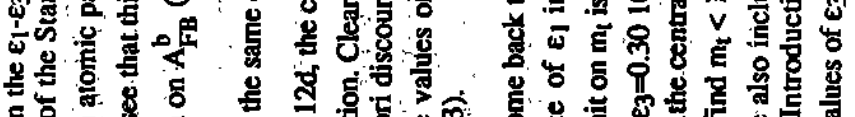

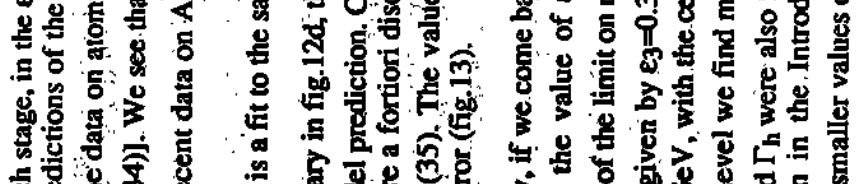

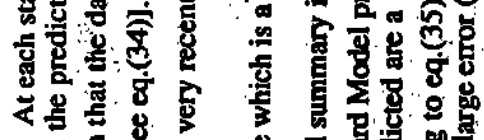

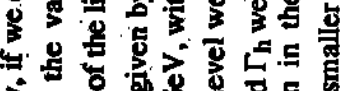

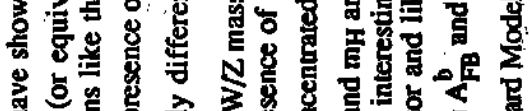

害密

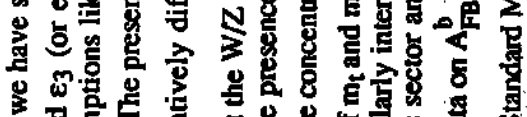

要

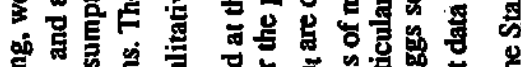

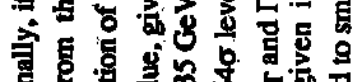

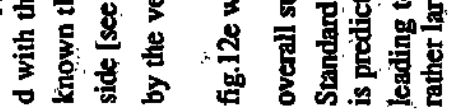

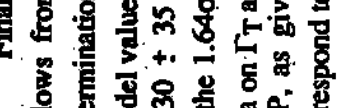

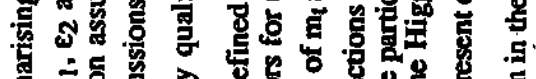

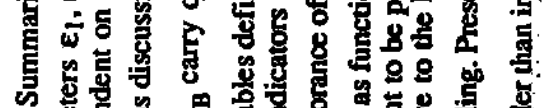

总产 

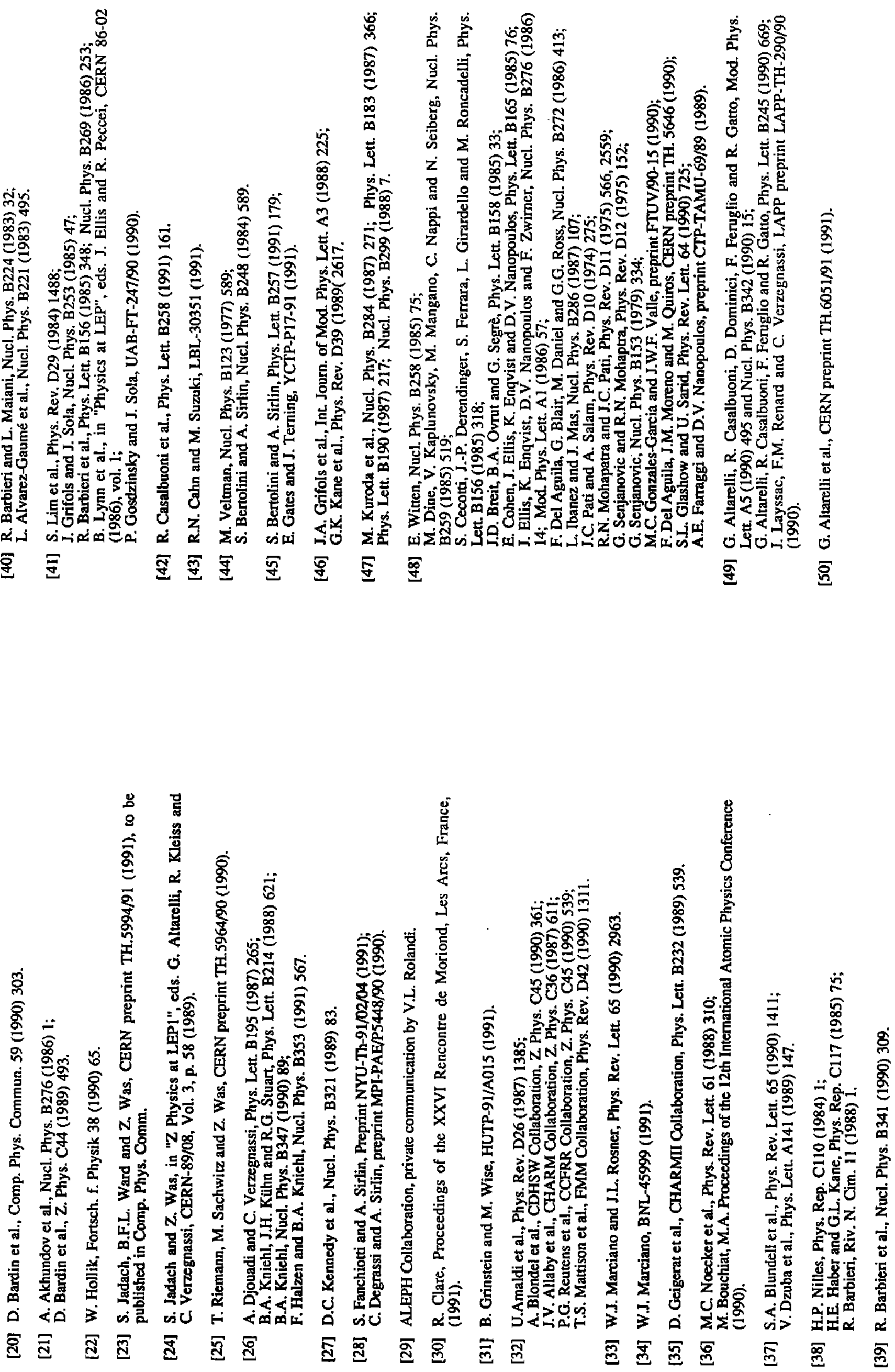


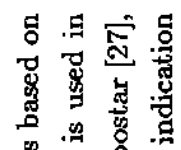

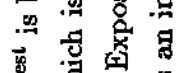

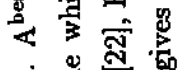

总苛善

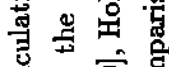

ฮี .동

焉兽

ก $\quad$ ง

旁

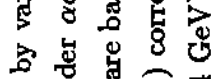

ช

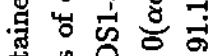

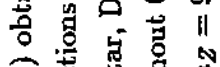

造密崂

通总

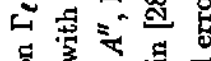

焉点

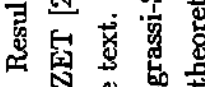

\begin{tabular}{|c|c|c|c|c|c|c|c|c|c|c|c|c|}
\hline ஜ̆ & & & & & & & 吕 & $\begin{array}{l}+ \\
\infty \\
\infty\end{array}$ & \begin{tabular}{|l|}
0 \\
$\ddot{\infty}$ \\
\end{tabular} & & & \\
\hline กิ & & & & & & & $\vec{\infty}$ & 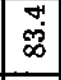 & \begin{tabular}{|l|} 
\\
$\infty$ \\
$\infty$
\end{tabular} & & & \\
\hline $\bar{n}$ & & & & & & & $\begin{array}{l}N \\
\infty \\
\infty\end{array}$ & $\stackrel{4}{\infty}$ & $\begin{array}{l}\dot{\infty} \\
\dot{\infty}\end{array}$ & & & \\
\hline 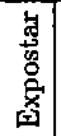 & 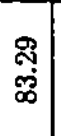 & 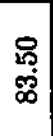 & $\begin{array}{l}\mathscr{2} \\
\infty \\
\infty\end{array}$ & 总 & 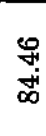 & $\begin{array}{l}\overrightarrow{0} \\
\dot{0} \\
\infty\end{array}$ & $\begin{array}{l}\tilde{\infty} \\
\infty \\
\infty\end{array}$ & $\begin{array}{l}\mathfrak{N} \\
\tilde{\infty} \\
\tilde{\infty}\end{array}$ & \begin{tabular}{|l}
$\mathscr{\infty}$ \\
$\mathscr{\infty}$ \\
$\infty$ \\
$\infty$
\end{tabular} & 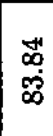 & 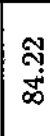 & 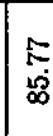 \\
\hline$\Sigma_{4}$ & $\begin{array}{l}\text { म̊ } \\
\infty \\
\infty\end{array}$ & 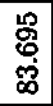 & \begin{tabular}{|l|}
$\stackrel{\varpi}{\infty}$ \\
$\infty$ \\
$\infty$
\end{tabular} & ঙุ円 & $\begin{array}{l}\overrightarrow{6} \\
\text { i }\end{array}$ & $\begin{array}{l}8 \\
8 \\
8\end{array}$ & 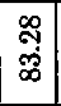 & 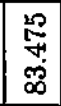 & $\begin{array}{l}\mathbb{N} \\
\infty \\
\infty\end{array}$ & $\begin{array}{l}\text { \% } \\
\text { के } \\
\end{array}$ & 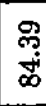 & $\begin{array}{l}0 \\
\infty \\
\infty \\
\infty\end{array}$ \\
\hline$\tau$ & $\begin{array}{l}\infty \\
\oplus \\
\infty \\
\infty\end{array}$ & 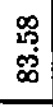 & \begin{tabular}{|l}
+ \\
$\infty$ \\
$\infty$ \\
$\infty$ \\
\end{tabular} & 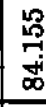 & $\begin{array}{l}\text { 施 } \\
\text { ד. } \\
\end{array}$ & 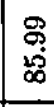 & $\begin{array}{l}0 \\
0 \\
\infty \\
\end{array}$ & \begin{tabular}{|l}
0 \\
$\infty$ \\
$\infty$ \\
$\infty$ \\
\end{tabular} & \begin{tabular}{|l}
$\overrightarrow{0}$ \\
$\ddot{\infty}$ \\
\end{tabular} & \begin{tabular}{|l|}
\multirow{\delta}{\infty}{} \\
$\tilde{\infty}$ \\
\end{tabular} & \begin{tabular}{|l} 
Aి \\
\$ే \\
\end{tabular} & 壳 \\
\hline$\frac{\overrightarrow{8}}{\nabla}$ & $\begin{array}{l}\infty \\
\infty \\
\infty \\
\infty\end{array}$ & $\begin{array}{l}\text { 莴 } \\
\infty \\
\infty\end{array}$ & $\underset{\infty}{F}$ & 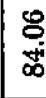 & 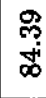 & $\begin{array}{l}\mathscr{8} \\
\stackrel{0}{0} \\
1\end{array}$ & $\begin{array}{c}m \\
\infty \\
\infty\end{array}$ & $\mid \begin{array}{l}2 \\
\tilde{m} \\
\tilde{\infty}\end{array}$ & 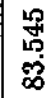 & $\begin{array}{l}\infty \\
\infty \\
\infty\end{array}$ & $\stackrel{0}{\stackrel{+}{+}}$ & 瓷 \\
\hline 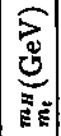 & ค8) & 요 & เి & 的 & คిస్ & เిల్లి & छ్.ి. & ర్రిశ్లి & క్రి & $1=$ & ర్రిల్తి & క్రిశ్ \\
\hline
\end{tabular}

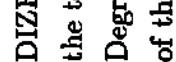

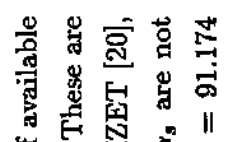

苍牙至

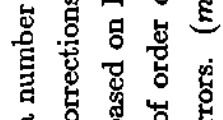

๘

今。

蒫密芯

卷卷吉

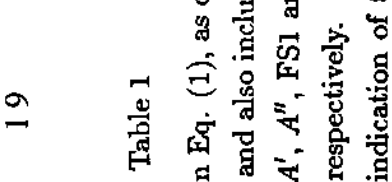

舟要

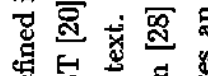

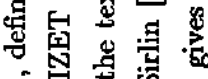

它吉焉

×

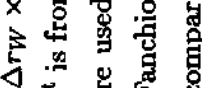

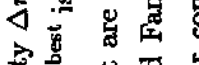

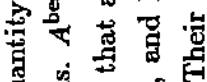

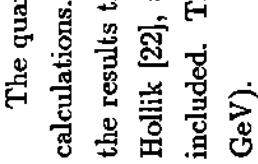

\begin{tabular}{|c|c|c|c|c|c|c|c|c|c|c|c|c|}
\hline 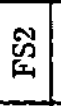 & $\begin{array}{c}\text { శ్ } \\
\text { ధ }\end{array}$ & $\begin{array}{l}20 \\
-7 \\
-1\end{array}$ & & $\stackrel{\vec{p}}{\not}$ & & $\begin{array}{l}\text { ન్ } \\
\overrightarrow{7} \\
\end{array}$ & & & & & & \\
\hline $\begin{array}{l}\overrightarrow{5} \\
\vec{\Sigma}\end{array}$ & $\begin{array}{l}\text { ज] } \\
\overline{1}\end{array}$ & 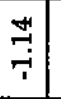 & & $\begin{array}{l}\stackrel{\leftrightarrow}{0} \\
\dot{p}\end{array}$ & & $\begin{array}{l}\text { สิ } \\
\overrightarrow{1}\end{array}$ & $\stackrel{\overbrace{}}{-}$ & 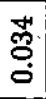 & & $\begin{array}{l}\text { ఝ్ } \\
\text { ه్ }\end{array}$ & & $\begin{array}{l}0 \\
\infty \\
0 \\
0\end{array}$ \\
\hline$\psi_{\psi}$ & $\begin{array}{l}9 \\
7 \\
\\
\end{array}$ & \begin{tabular}{l}
9 \\
\hdashline \\
$i$
\end{tabular} & $\begin{array}{l}\text { ్ָ } \\
\text { ฟิ }\end{array}$ & $\begin{array}{l}\nexists ⿱ 乛 \\
\stackrel{p}{p}\end{array}$ & 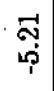 & 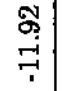 & $\begin{array}{l}\stackrel{⿰}{\circ} \\
\stackrel{+}{+}\end{array}$ & 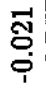 & $\begin{array}{l}z \\
i \\
i\end{array}$ & $\begin{array}{l}\stackrel{\mathscr{m}}{\mathrm{j}} \\
\text { }\end{array}$ & $\begin{array}{l}\mathcal{N} \\
\stackrel{\gamma}{p}\end{array}$ & 孚 \\
\hline ; & $\begin{array}{l}2 \\
2 \\
0 \\
1\end{array}$ & בְ & 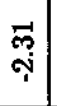 & 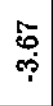 & ํㅗㅇ & $\begin{array}{l}\vec{\sigma} \\
\overrightarrow{1} \\
\overrightarrow{1}\end{array}$ & $\begin{array}{l}\stackrel{8}{-} \\
+\end{array}$ & 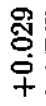 & \begin{tabular}{c}
8 \\
\hdashline \\
\hdashline
\end{tabular} & 苟 & 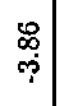 & $\begin{array}{l}20 \\
\stackrel{0}{0} \\
\end{array}$ \\
\hline 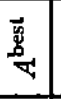 & $\begin{array}{l}7 \\
0 \\
0 \\
\end{array}$ & \begin{tabular}{l}
8 \\
\hdashline \\
1
\end{tabular} & $\begin{array}{l}8 \\
\text { ণิ }\end{array}$ & 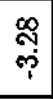 & 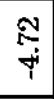 & 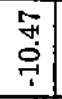 & $\begin{array}{l}9 \\
\stackrel{9}{7} \\
+\end{array}$ & $\begin{array}{l}\mathscr{\infty} \\
\stackrel{+}{+} \\
+\end{array}$ & $\begin{array}{c}\overrightarrow{0} \\
0 \\
i \\
i\end{array}$ & 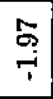 & 苛 & $\begin{array}{l}9 \\
0 \\
0\end{array}$ \\
\hline 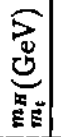 & గొ৪) & ธิ & หู่ & in & Вస్ & చి్ㅣ & & ל్t: & క్రి్․ & క్రిల & & ప్రిం్లి \\
\hline
\end{tabular}




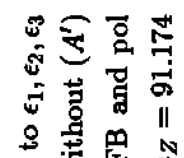

岁茎 罢

炭它总

过 要

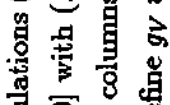

矢娄

ฮै है

安客岁素

究

要总总客

\begin{tabular}{|c|c|c|c|c|c|c|c|c|c|c|c|c|}
\hline & 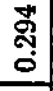 & $\begin{array}{r}8 \\
0 \\
\vdots \\
0 \\
\end{array}$ & 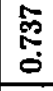 & Fị: & 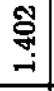 & 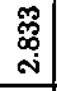 & \begin{tabular}{|l}
5 \\
0 \\
0 \\
\end{tabular} & 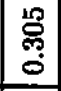 & 号 & $\begin{array}{l}8 \\
\infty \\
0 \\
\end{array}$ & ָָ ָָ & \\
\hline & 0 & 㤎 & 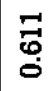 & $\mathscr{\varpi}$ & $\underset{-}{\stackrel{D}{-}}$ & $\begin{array}{l}: \\
\stackrel{8}{*} \\
\text { s. }\end{array}$ & 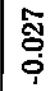 & 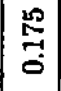 & 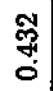 & $\stackrel{2}{\stackrel{2}{5}}$ & 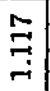 & \\
\hline & & 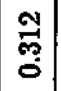 & 哭 & & & 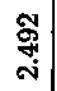 & 总 & శ్త్ & $\begin{array}{l}\widetilde{Ð} \\
0\end{array}$ & $\begin{array}{l}\text { 莒 } \\
\stackrel{\circ}{\circ}\end{array}$ & $\vec{\sigma}$ & 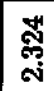 \\
\hline $\mathrm{i}_{\mathrm{i}}$ & 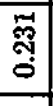 & 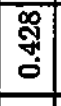 & $\begin{array}{l}\infty \\
0 \\
0 \\
0 \\
0\end{array}$ & $\Phi$ & 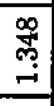 & 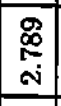 & \begin{tabular}{|l|} 
so \\
o \\
0
\end{tabular} & 寻 & 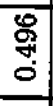 & 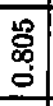 & & \\
\hline & $\begin{array}{l}0 \\
0 \\
0\end{array}$ & 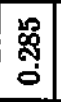 & $\overrightarrow{7}$ & & : & 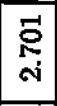 & $\begin{array}{l}\text { 蒙 } \\
\end{array}$ & $\begin{array}{c}\text { 号 } \\
\vdots \\
\end{array}$ & 0 & $\begin{array}{l}\mathbf{N} \\
0 \\
0 \\
0\end{array}$ & & 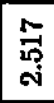 \\
\hline & 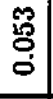 & : ํํㅇ & & & & $\begin{array}{l}z \\
7 \\
\text { in }\end{array}$ & $\mathscr{\pi}$ & & & 9 & & \\
\hline$\xi$ & & 웜 & 吅高 & $s=1$ & 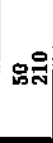 & काल & (985 & & $\Rightarrow 4$ & $=0$ & : & \\
\hline
\end{tabular}

曲焉焉

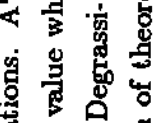

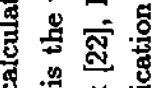

卷

找和

胥空

क

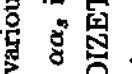

点口

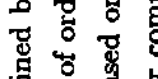

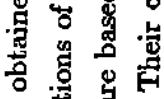

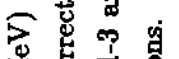

\&

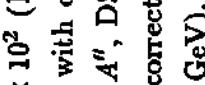

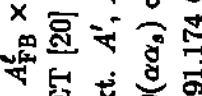

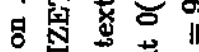

巻宫焉总

灵吕吉焉

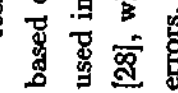

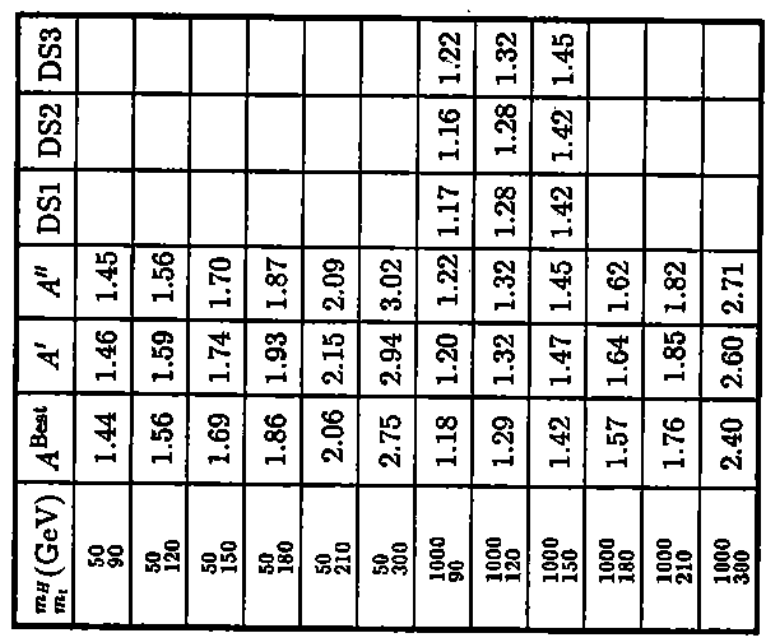




\begin{tabular}{|c|c|c|c|c|c|c|c|c|c|c|c|c|}
\hline$=\overline{8}$ & & \begin{tabular}{l}
0 \\
\multirow{2}{*}{} \\
0 \\
\end{tabular} & $\begin{array}{l}\vec{Z} \\
\vdots \\
0\end{array}$ & 㣽 & 0 & & $\begin{array}{l}20 \\
20 \\
0 \\
\end{array}$ & ס్ & 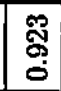 & 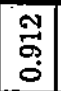 & 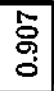 & \\
\hline$=$ & & \begin{tabular}{l}
\multirow{0}{0}{} \\
\\
\end{tabular} & $\begin{array}{l}8 \\
\text { o. } \\
0 \\
\end{array}$ & 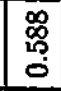 & 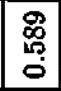 & & 哭 & 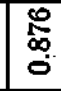 & $\begin{array}{l}\overrightarrow{0} \\
0 \\
0\end{array}$ & \begin{tabular}{|c|} 
\\
\\
0 \\
0 \\
0
\end{tabular} & & \\
\hline $\overrightarrow{8} \bar{g}$ & & 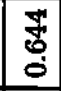 & \begin{tabular}{|l|}
\multirow{0}{*}{} \\
0 \\
\end{tabular} & \begin{tabular}{|l} 
\\
0 \\
0 \\
0
\end{tabular} & 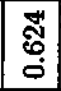 & & & 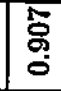 & 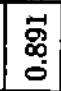 & & & \\
\hline$=0^{\infty}$ & ฟ & \begin{tabular}{|l}
\multirow{3}{*}{} \\
: \\
\end{tabular} & \begin{tabular}{|c|}
\multirow{2}{*}{} \\
$\stackrel{9}{0}$ \\
\end{tabular} & $\begin{array}{l}0 \\
\vdots \\
0\end{array}$ & & & & \begin{tabular}{|l}
20 \\
0 \\
0 \\
0
\end{tabular} & 范 & & & \\
\hline$t$ & 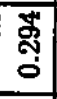 & \begin{tabular}{|c|}
\multirow{2}{*}{} \\
\\
\end{tabular} & \begin{tabular}{|l|}
\multirow{2}{*}{} \\
0 \\
\end{tabular} & \begin{tabular}{|l}
\multirow{2}{*}{} \\
ज़ \\
\end{tabular} & 는 & & & 㞔 & & & & \\
\hline & ఫ్లె' & 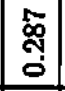 & $\overrightarrow{\mathbf{D}}$ & $\Phi$ & & & & & & & & \\
\hline & & & & & & & & & & & & \\
\hline
\end{tabular}

\begin{tabular}{|c|c|c|c|c|c|c|c|c|c|c|c|c|}
\hline & 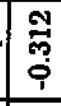 & \begin{tabular}{|c} 
\\
Y. \\
\\
\end{tabular} & \begin{tabular}{|c}
$\mathscr{0}$ \\
0 \\
0 \\
\end{tabular} & 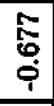 & $\overrightarrow{0}$ & 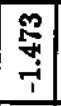 & \begin{tabular}{|c|} 
\\
0 \\
0 \\
\end{tabular} & $\begin{array}{c} \\
0 \\
0 \\
0 \\
\end{array}$ & 0 & & 苫 & \\
\hline & $\stackrel{4}{7}$ & \begin{tabular}{|c|}
$\vec{y}$ \\
0 \\
\end{tabular} & \& & & & 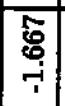 & $\begin{array}{l}0 \\
0 \\
0 \\
0\end{array}$ & \begin{tabular}{|l}
8 \\
\end{tabular} & & & & \\
\hline & 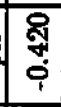 & $\begin{array}{l} \\
\text { 是 } \\
0 \\
0\end{array}$ & & & & 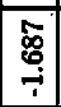 & 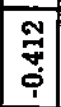 & & & & \begin{tabular}{|l|l}
\multirow{2}{0}{} \\
$\vdots$ \\
0
\end{tabular} & \\
\hline & $\begin{array}{l}\overrightarrow{3} \\
\\
\end{array}$ & N & & & & \begin{tabular}{|c|}
\multirow{2}{:}{} \\
$\rightarrow$ \\
\end{tabular} & 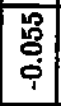 & \begin{tabular}{|l|}
8 \\
\end{tabular} & & & & \\
\hline & ত্⿳亠丷厂心 & $\infty$ & & & & 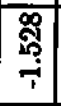 & \begin{tabular}{|c|}
9 \\
0 \\
0 \\
1
\end{tabular} & & & & \begin{tabular}{|l|} 
\\
\\
0 \\
0
\end{tabular} & \\
\hline & 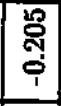 & & & & & & & & & & 0 & \\
\hline & & & & & & & & & & & క్రిశ్సి & \\
\hline
\end{tabular}




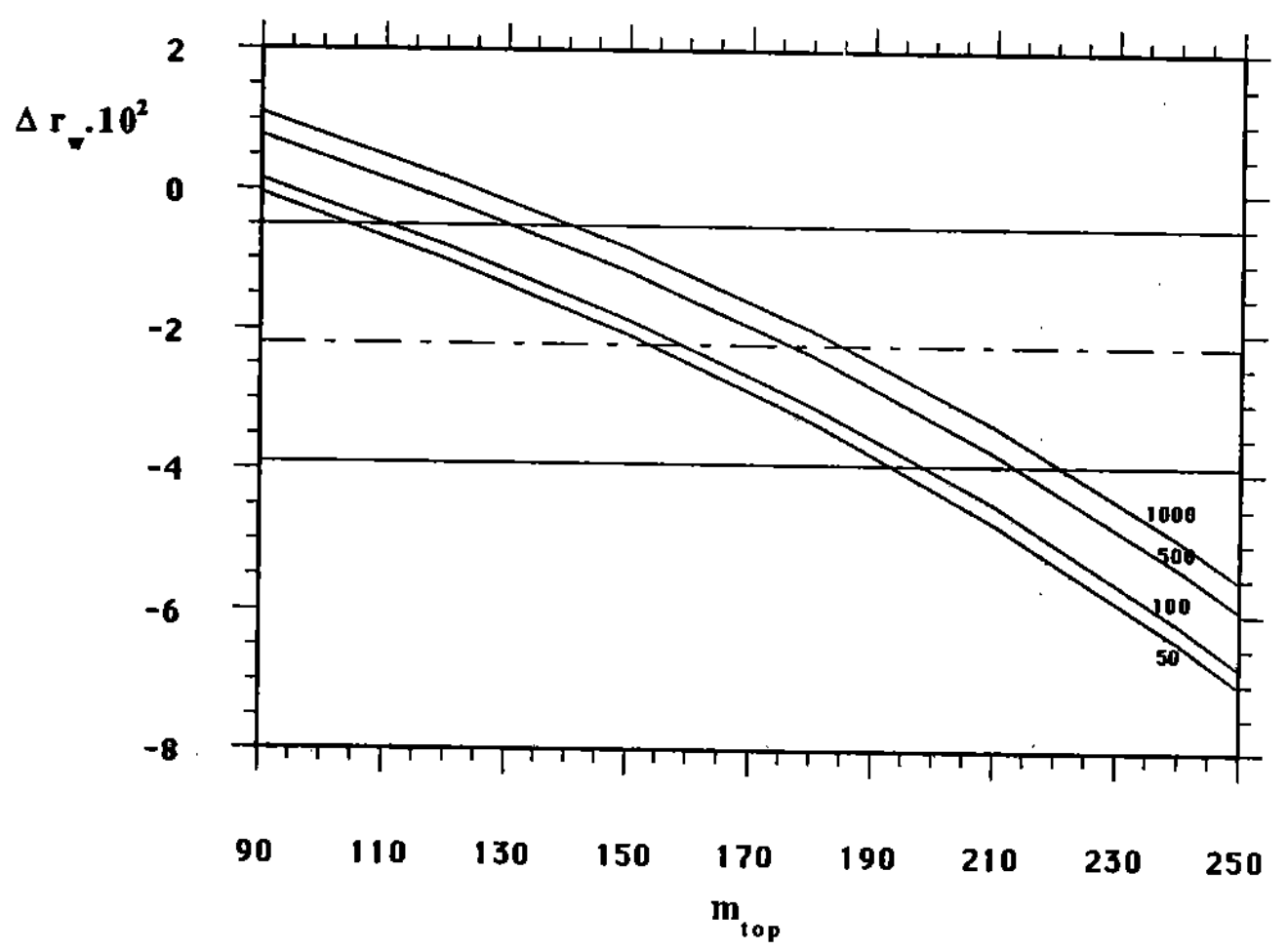

FIGURE 1

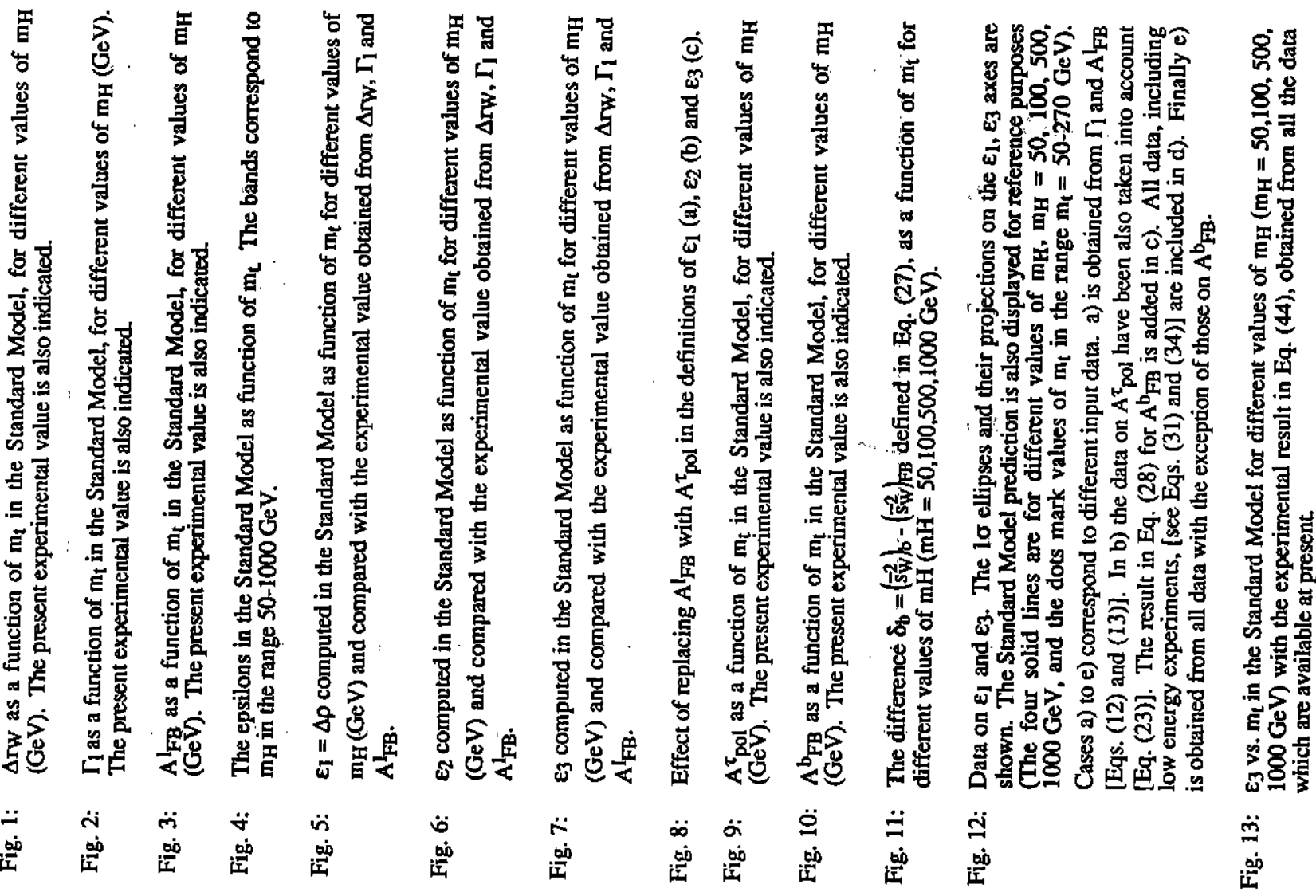




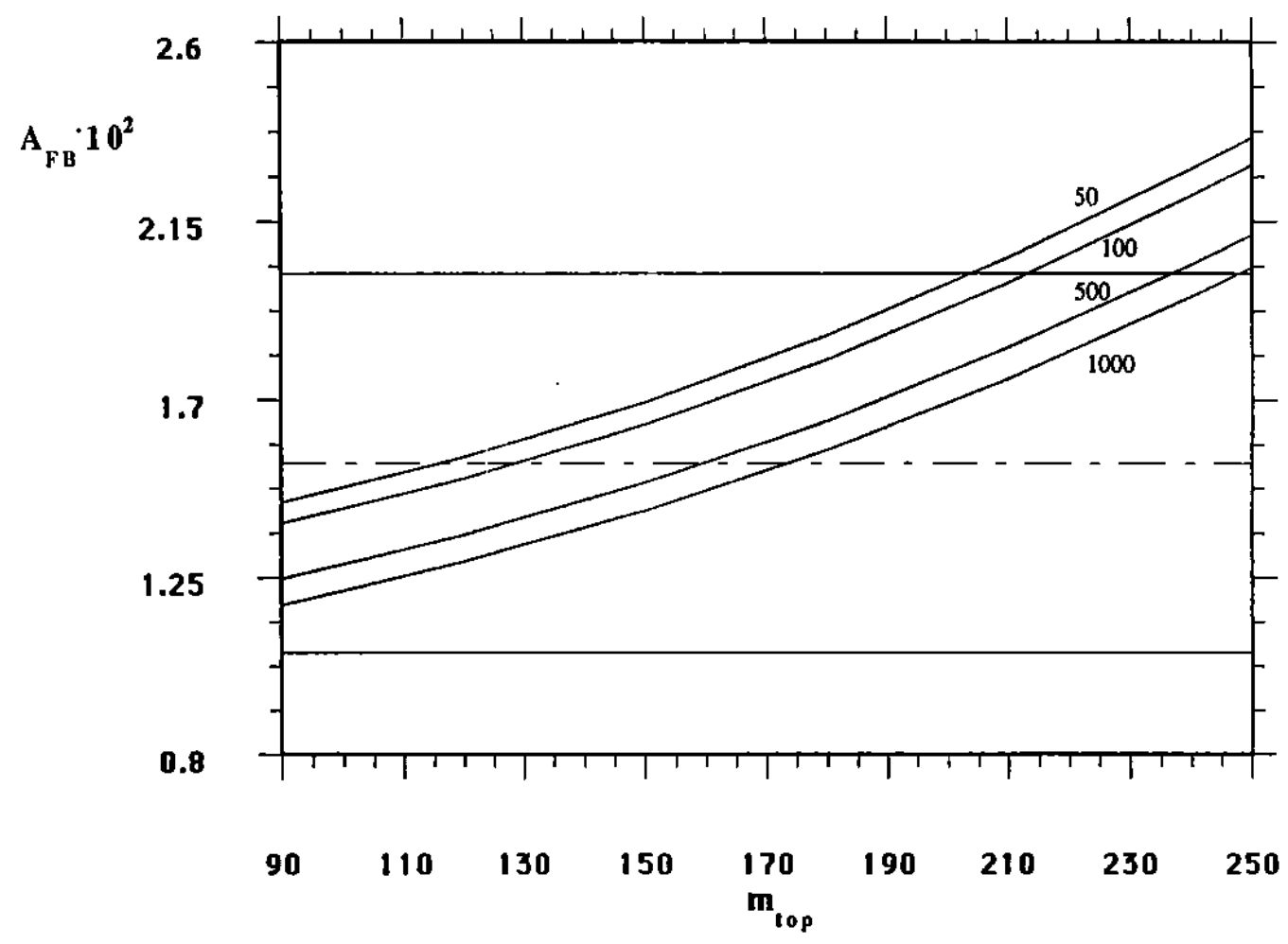

FIGURE 3

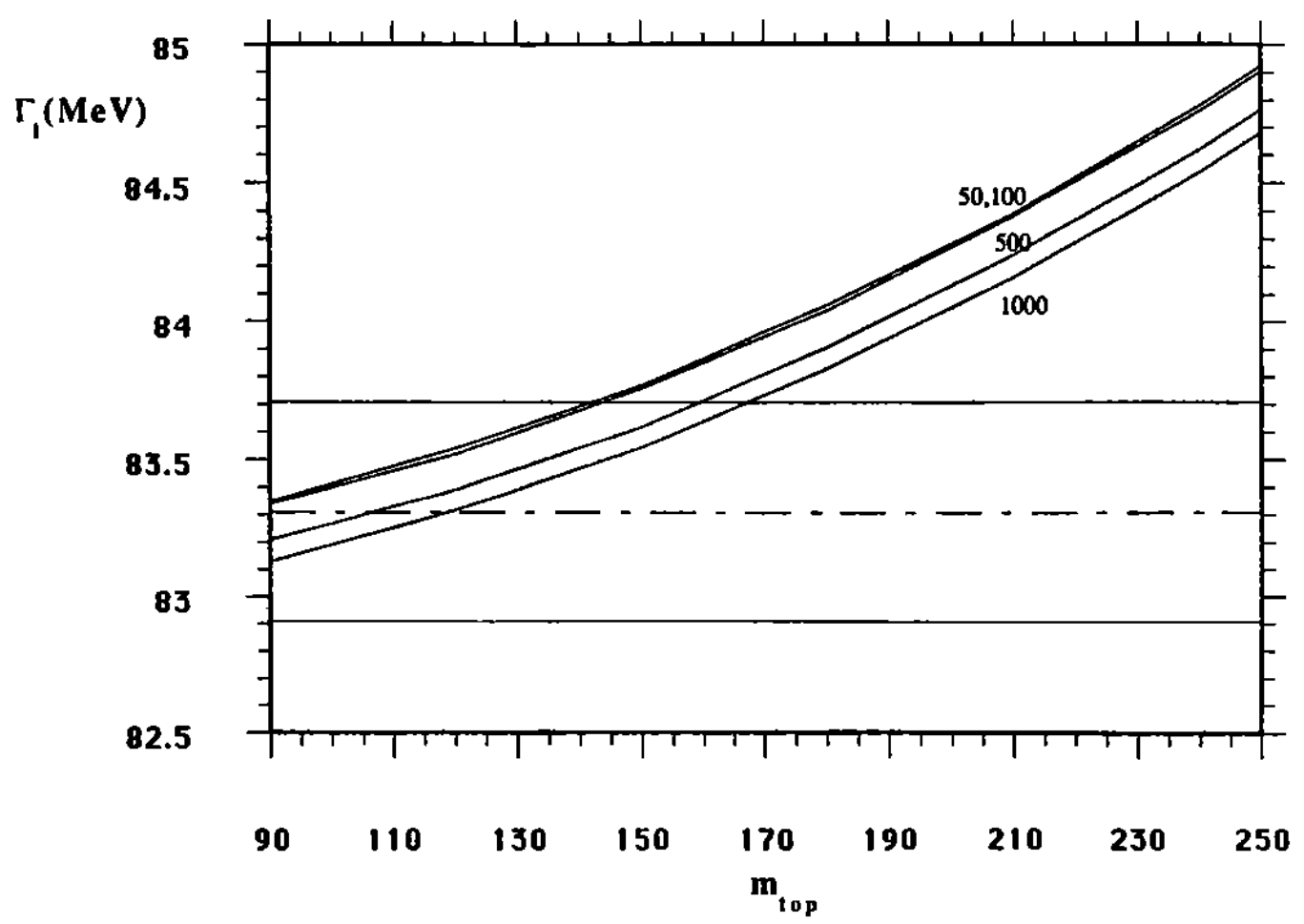

FIGURE 2 


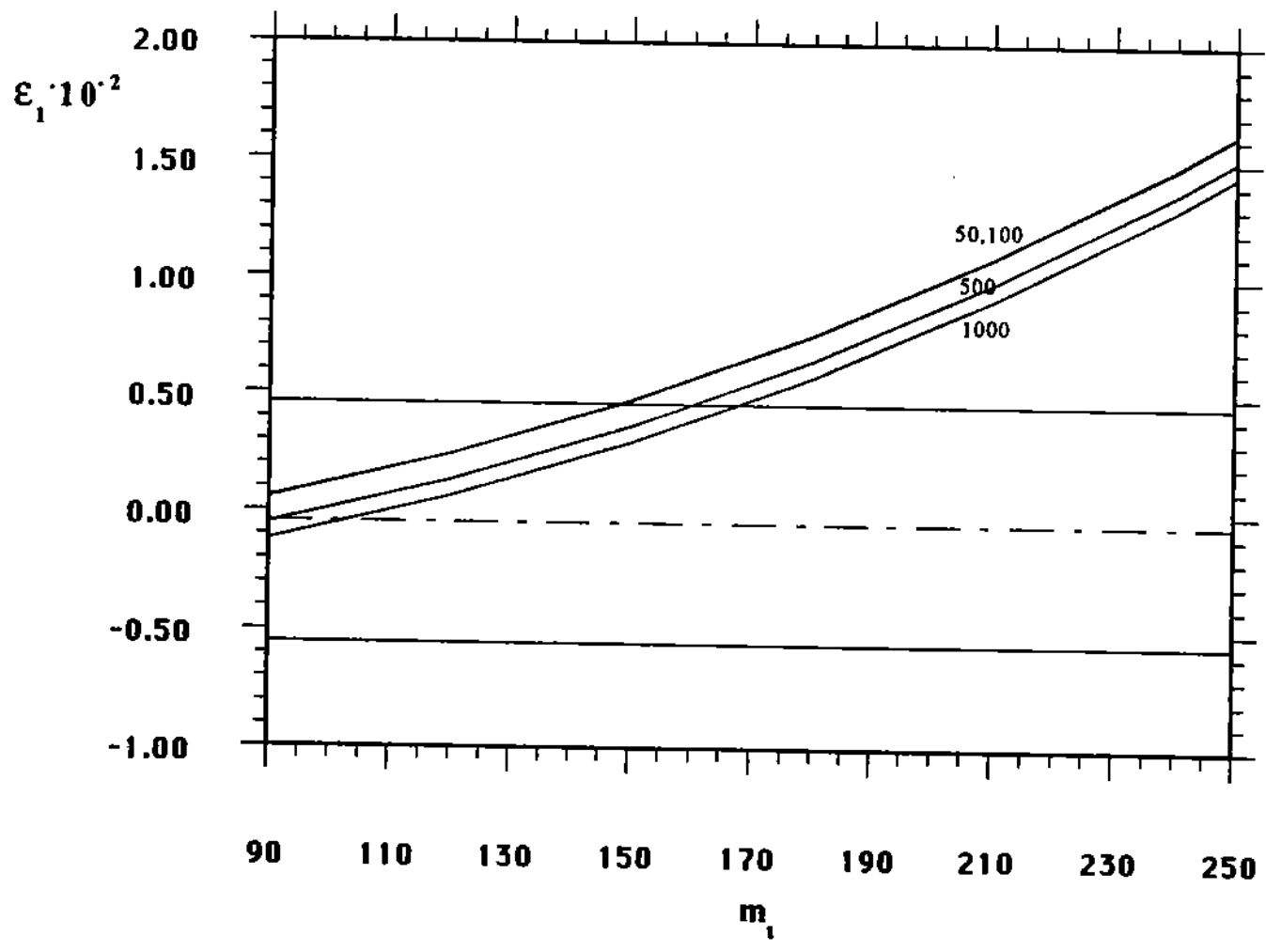

FIGURE 5

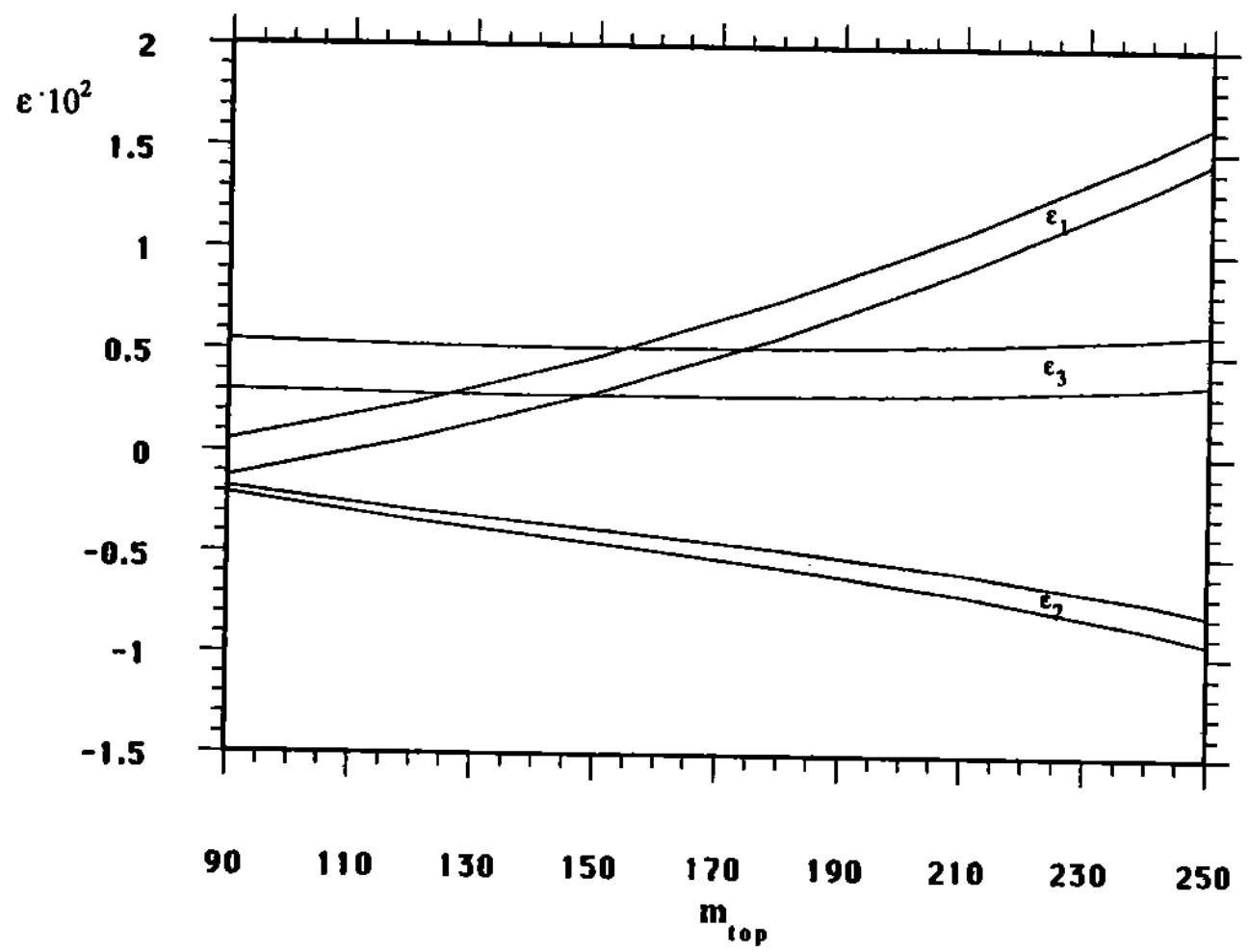

FIGURE 4 


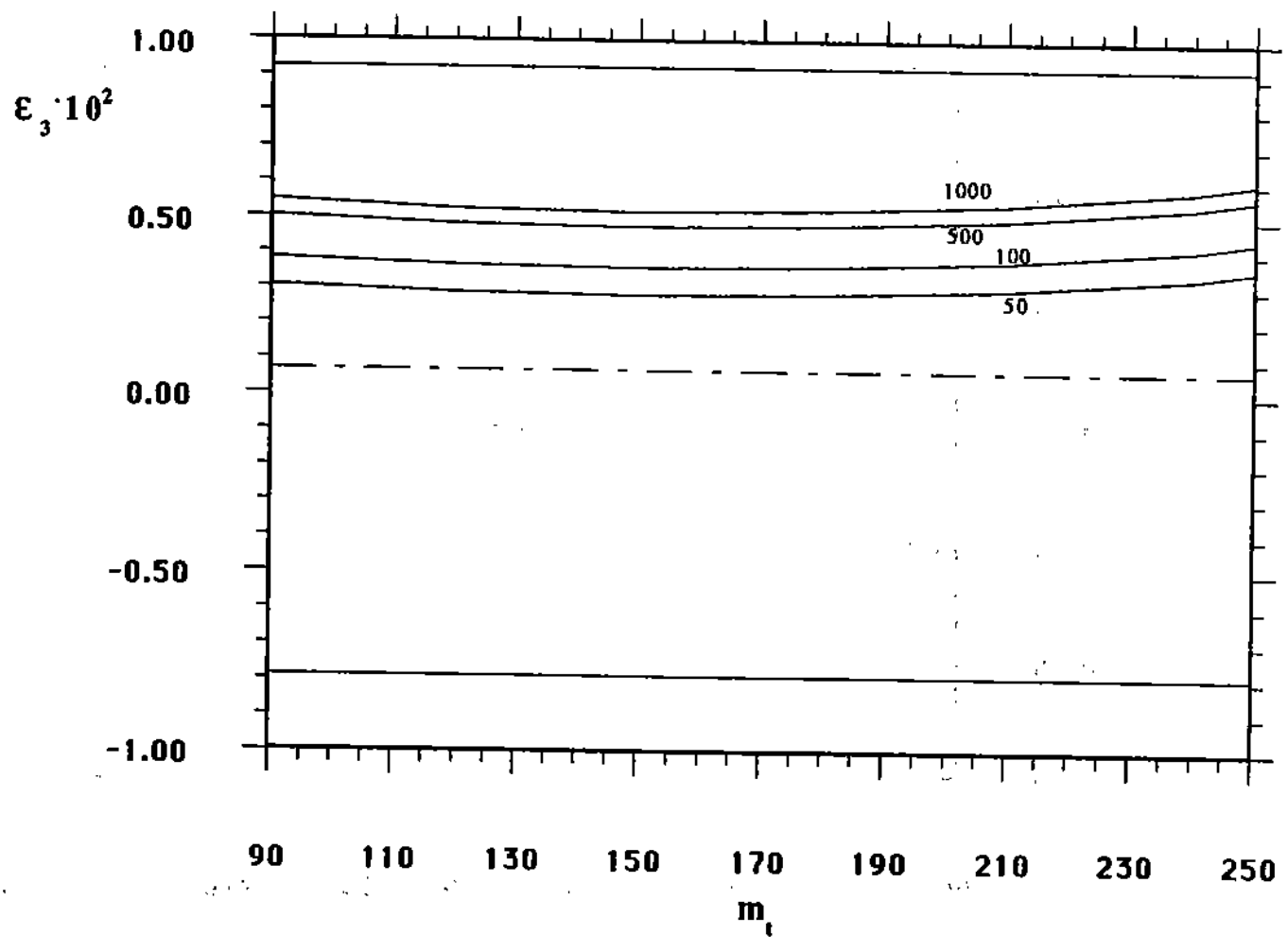

FIGURE 7

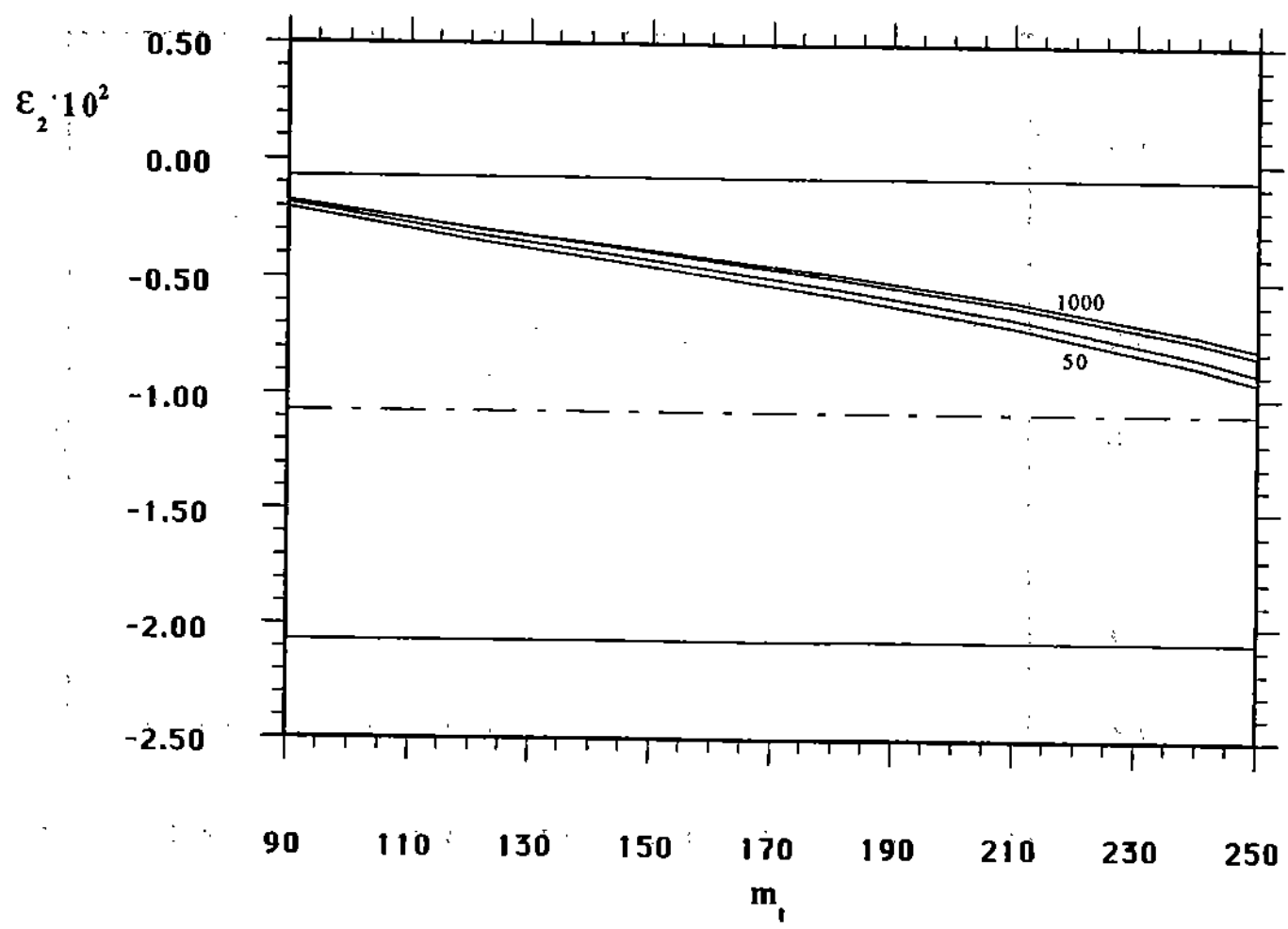

FIGURE 6 


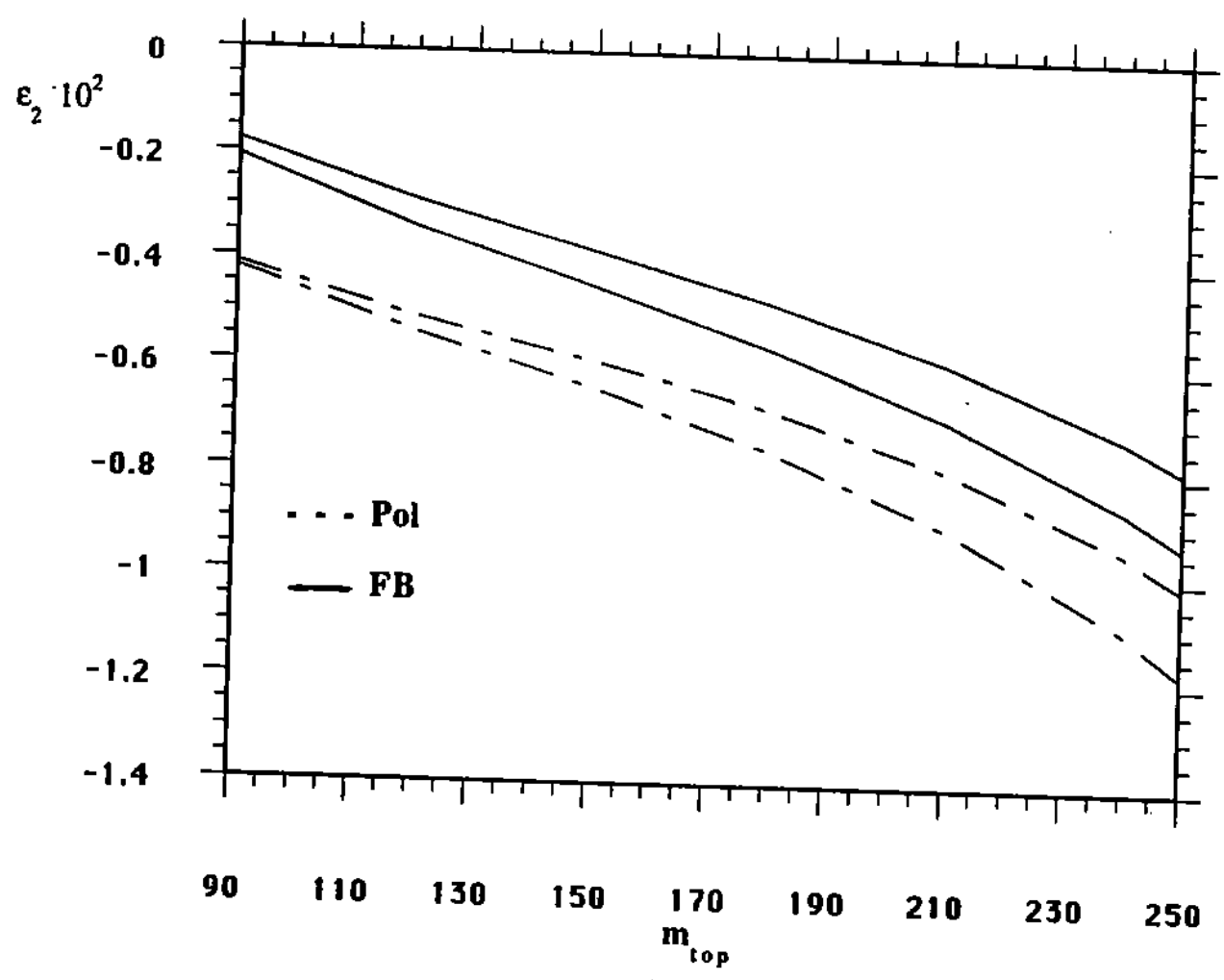

FIGURE $\mathrm{Bb}$

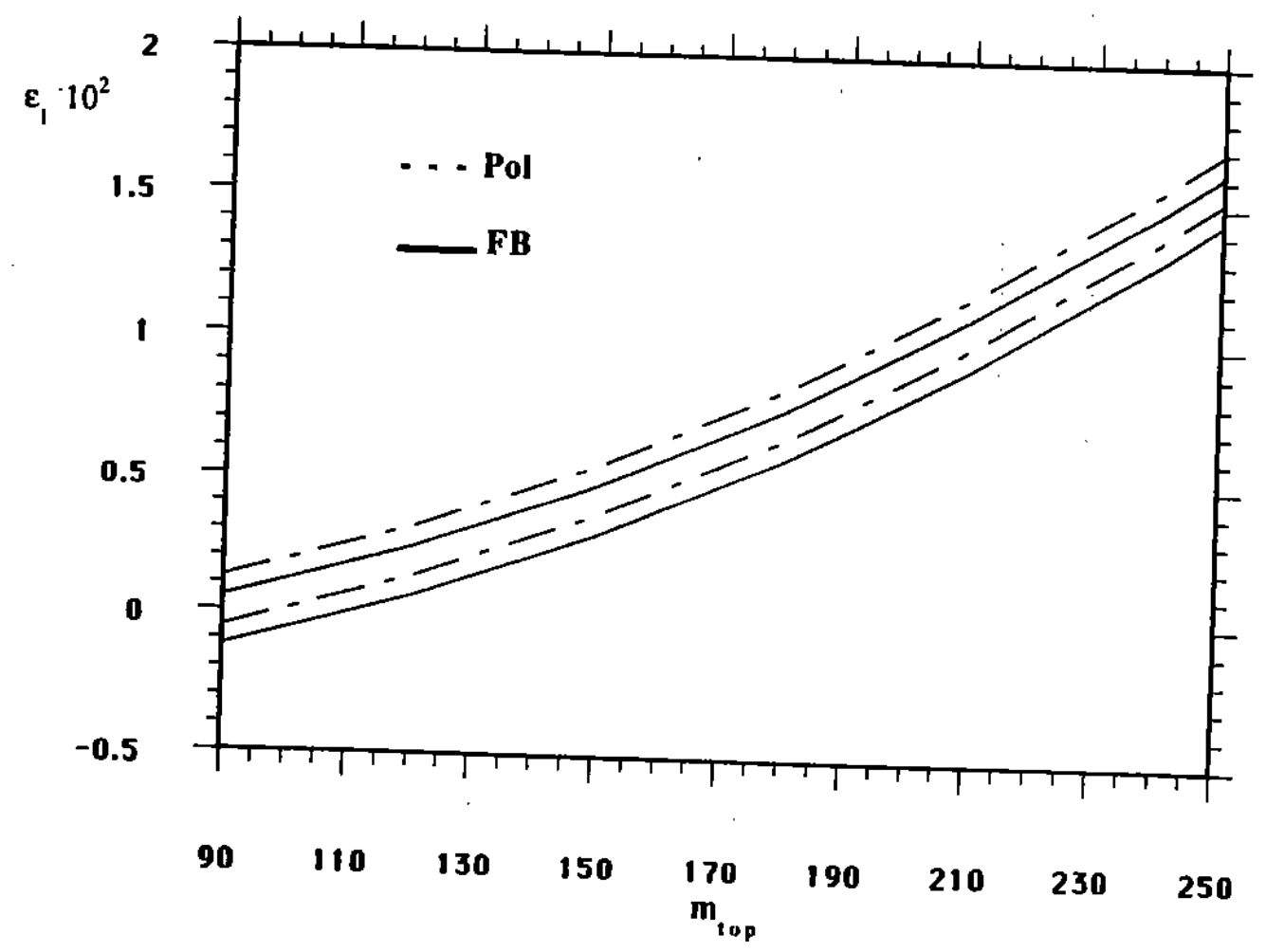

FIGURE $8 \mathrm{a}$ 


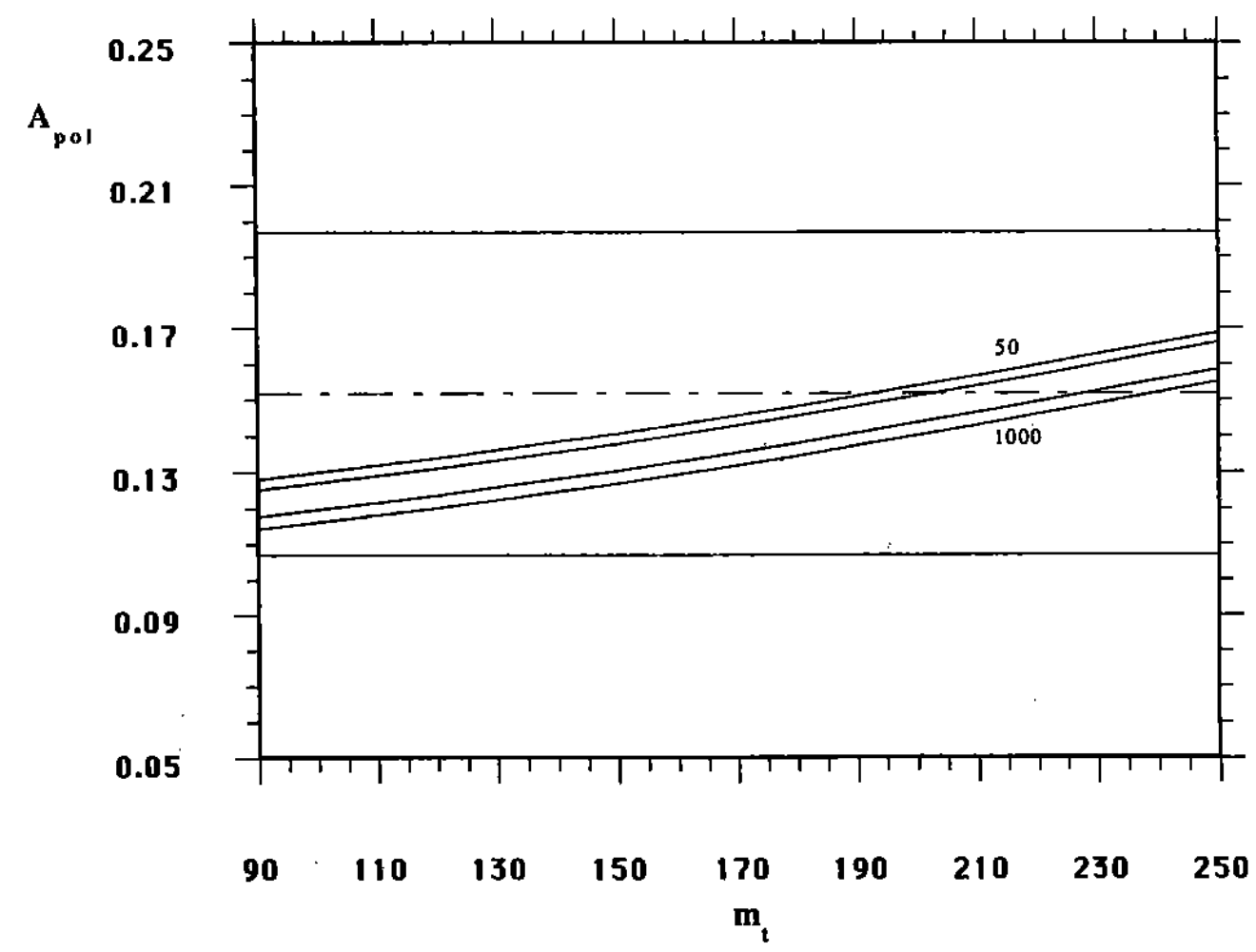

FITURE 9

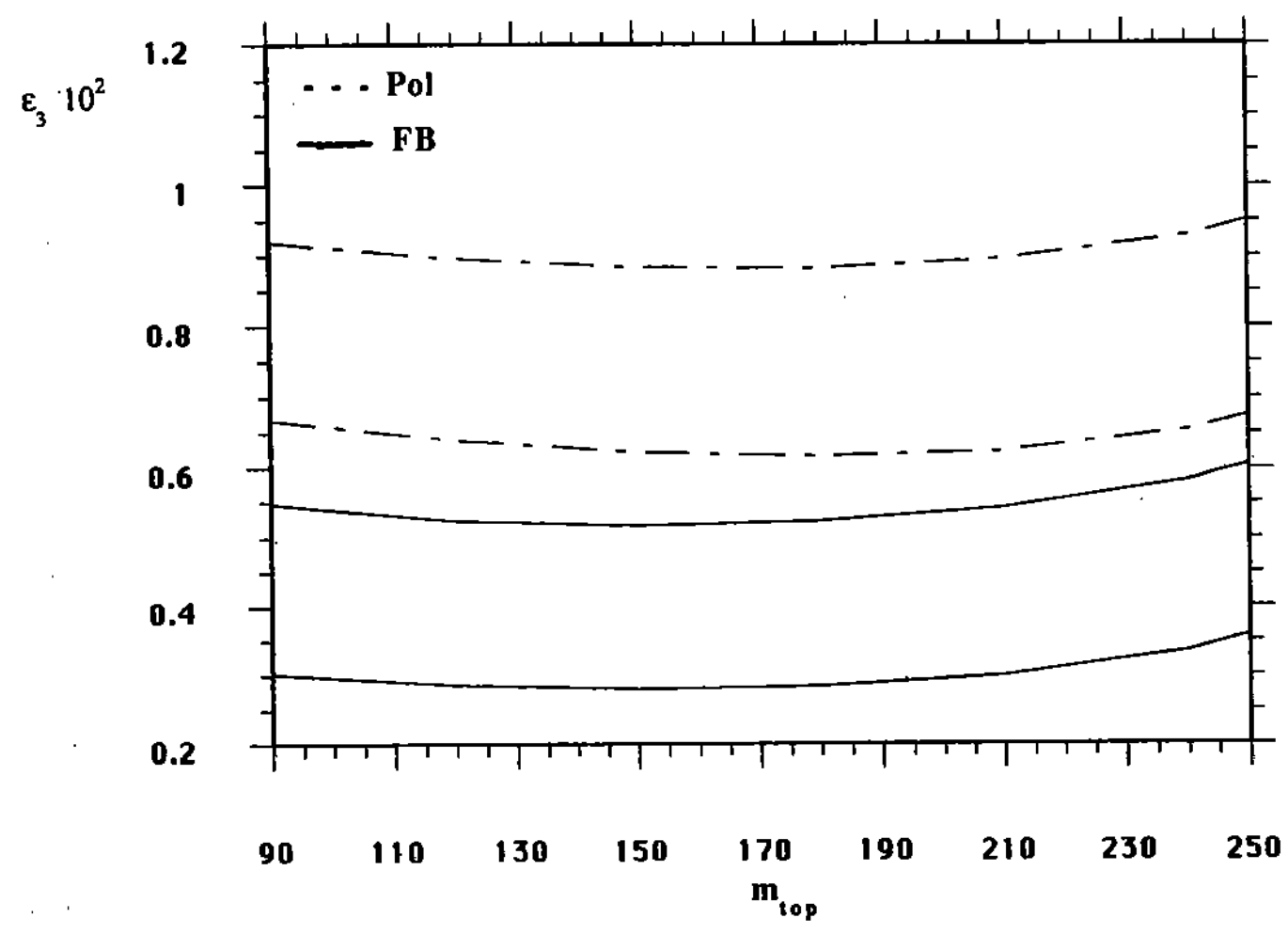

FIGURE BC 


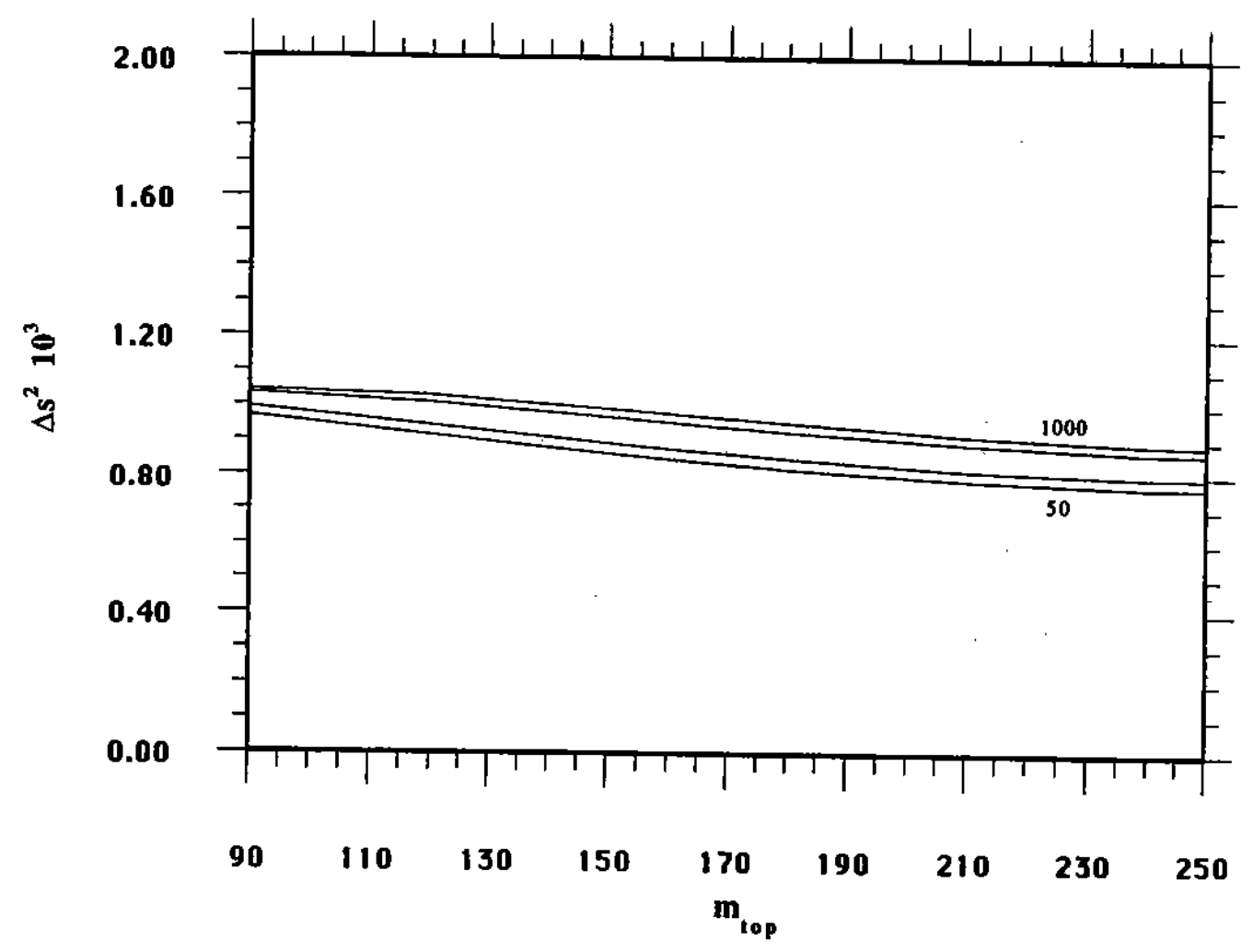

FIGURE 11

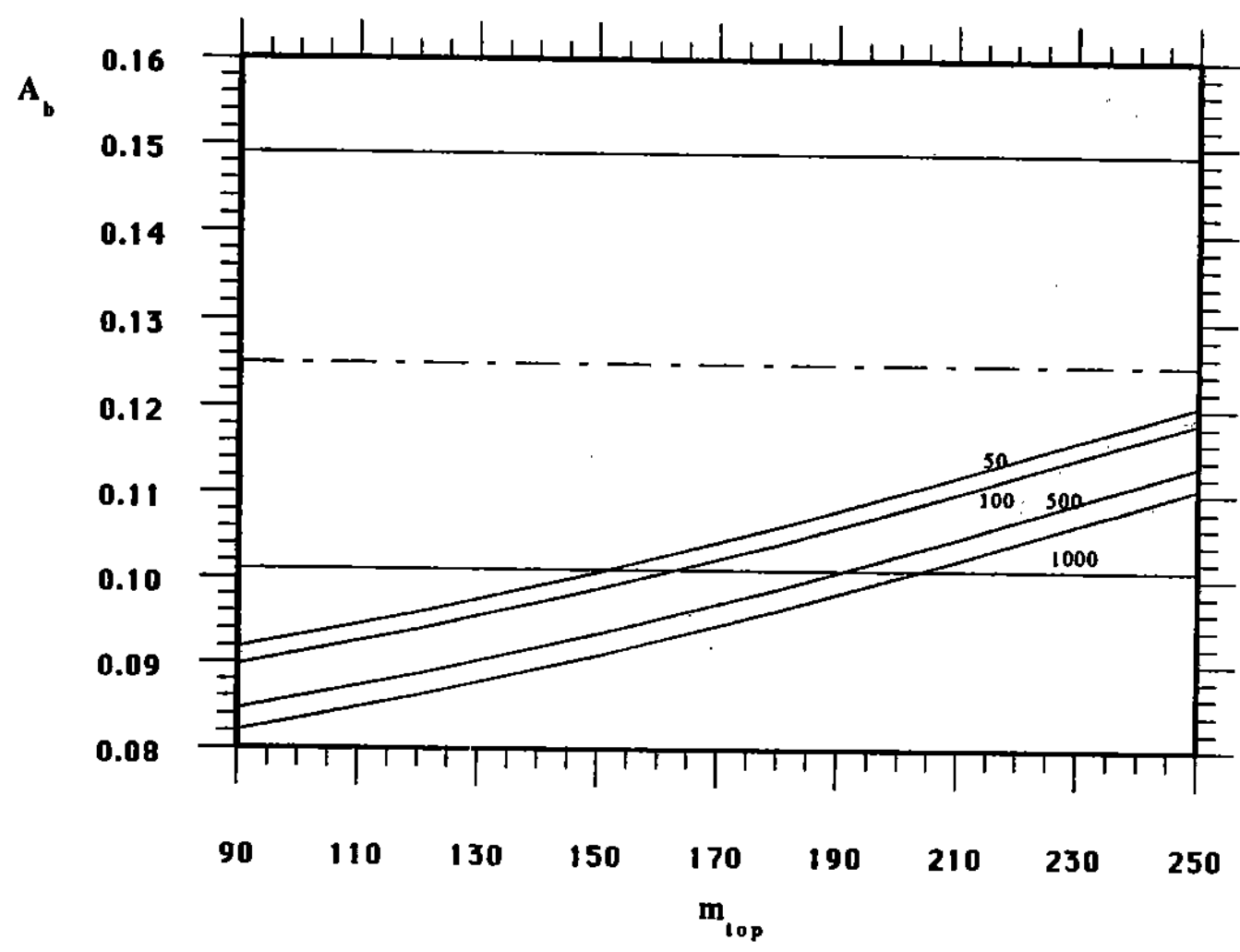

FIGURE 10 

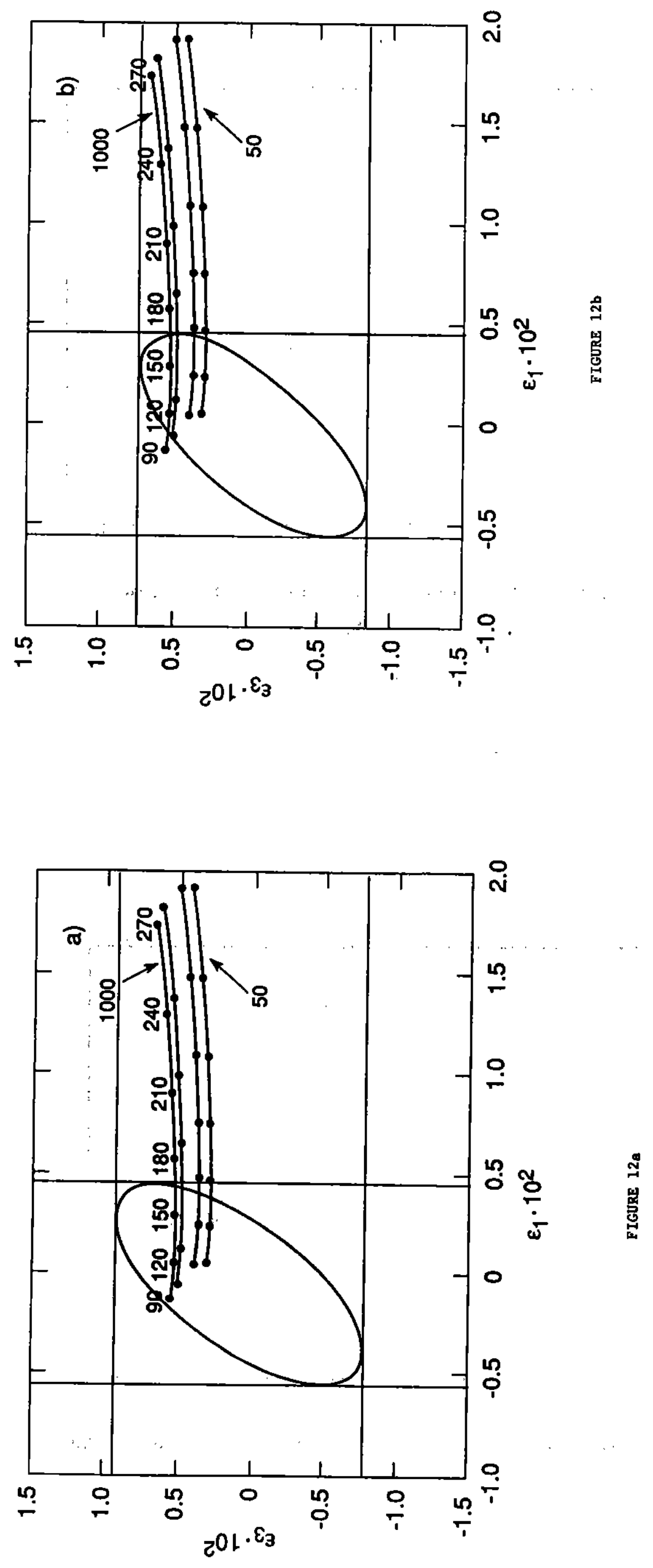

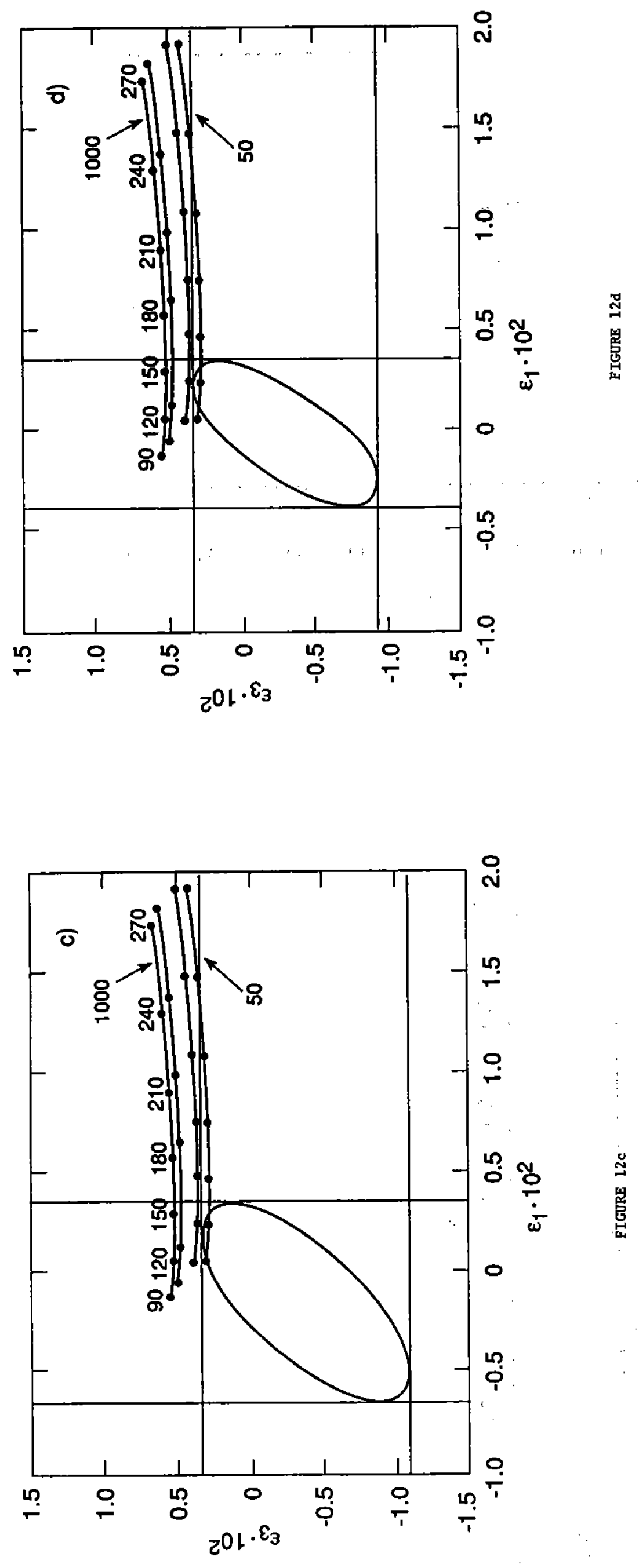


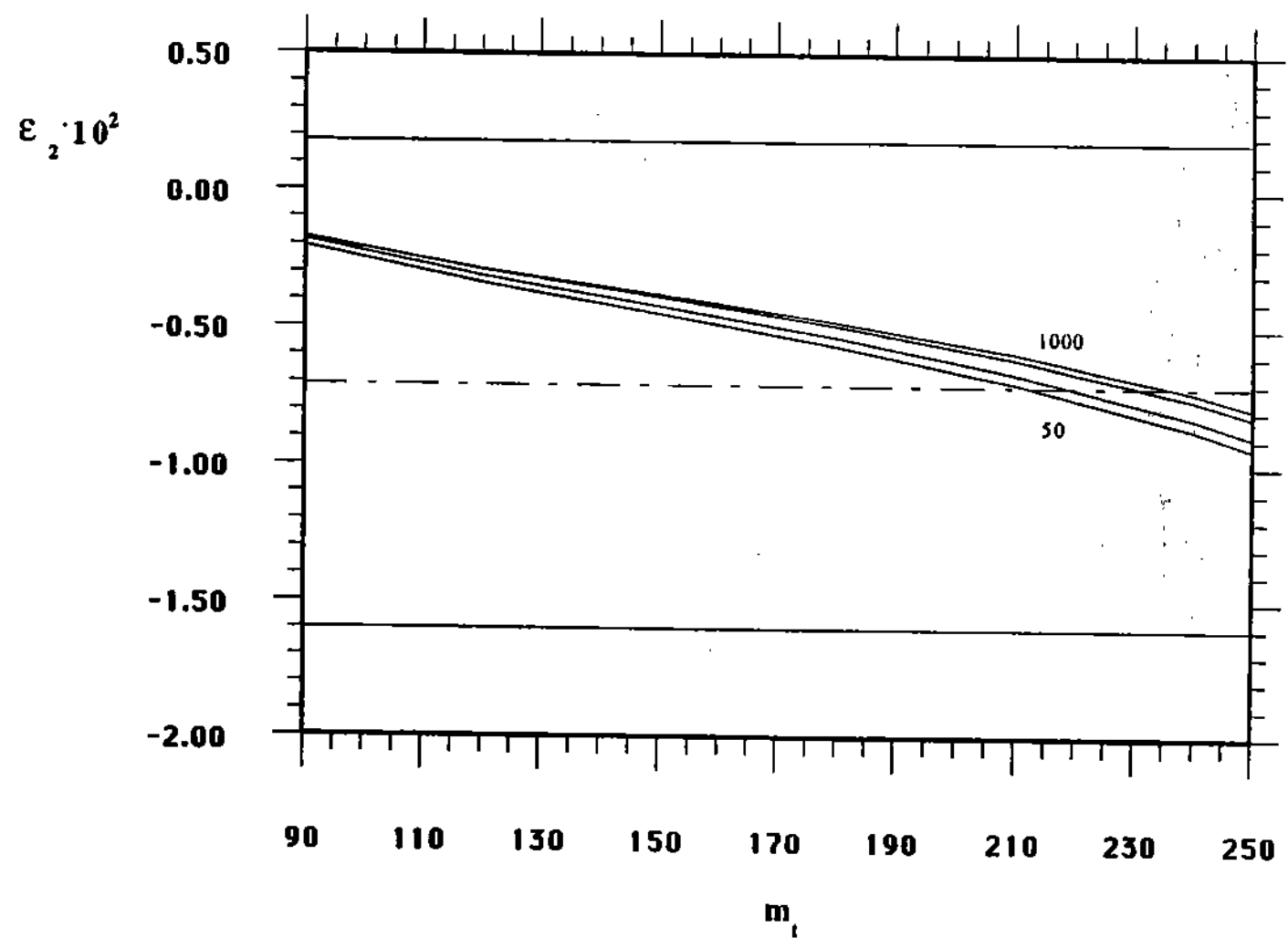

FIGURE 13

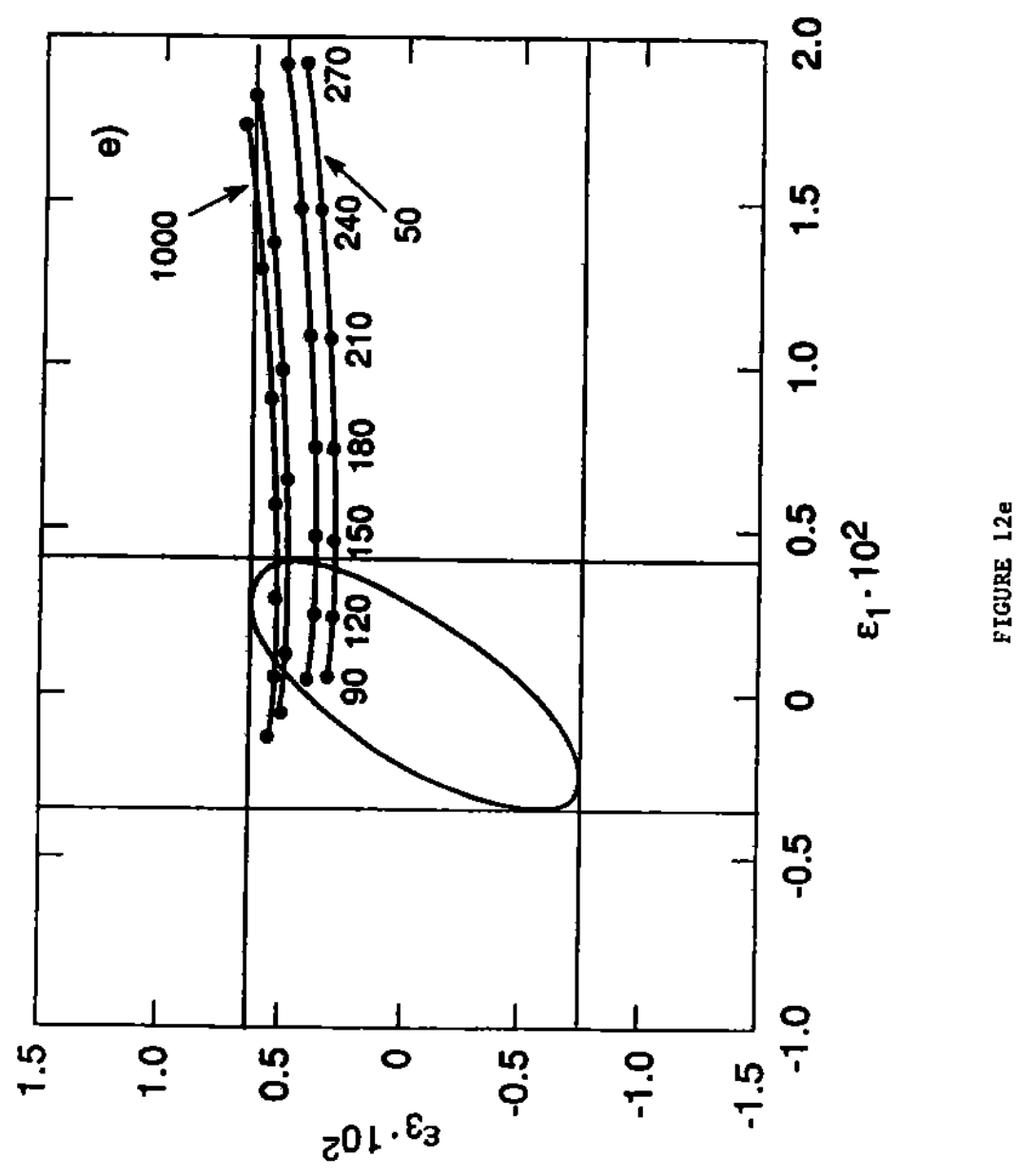

